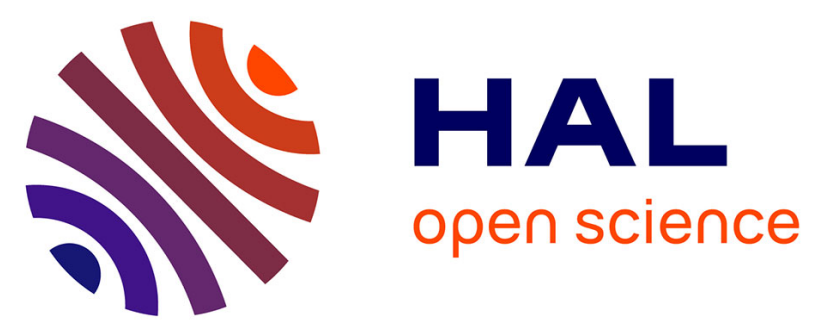

\title{
Prospection et inventaire archéologiques dans la vallée du Gökırmak. Premier rapport préliminaire: région de Taşköprü
}

\author{
Claire Barat, Julie Dalaison, Fabrice Delrieux, Jean-François Pichonneau, \\ Bernard Rémy
}

\section{To cite this version:}

Claire Barat, Julie Dalaison, Fabrice Delrieux, Jean-François Pichonneau, Bernard Rémy. Prospection et inventaire archéologiques dans la vallée du Gökırmak. Premier rapport préliminaire: région de Taşköprü. Anatolia antiqua = Eski anadolu / recueil de travaux publiés par l'Institut français d'études anatoliennes d'Istanbul , 2009, 17, pp.393 - 409. 10.3406/anata.2009.1294 . halshs-01429174

\section{HAL Id: halshs-01429174 \\ https://shs.hal.science/halshs-01429174}

Submitted on 7 Jan 2017

HAL is a multi-disciplinary open access archive for the deposit and dissemination of scientific research documents, whether they are published or not. The documents may come from teaching and research institutions in France or abroad, or from public or private research centers.
L'archive ouverte pluridisciplinaire HAL, est destinée au dépôt et à la diffusion de documents scientifiques de niveau recherche, publiés ou non, émanant des établissements d'enseignement et de recherche français ou étrangers, des laboratoires publics ou privés. 


\section{Prospection et inventaire archéologiques dans la vallée du} Gökırmak. Premier rapport préliminaire : région de Taşköprü

Claire Barat, Julie Dalaison, Fabrice Delrieux, Jean-François Pichonneau, Bernard Rémy

\section{Citer ce document / Cite this document :}

Barat Claire, Dalaison Julie, Delrieux Fabrice, Pichonneau Jean-François, Rémy Bernard. Prospection et inventaire archéologiques dans la vallée du Gökırmak. Premier rapport préliminaire : région de Taşköprü. In: Anatolia Antiqua, Tome 17, 2009. pp. 393-409.

doi : 10.3406/anata.2009.1294

http://www.persee.fr/doc/anata_1018-1946_2009_num_17_1_1294

Document généré le 09/09/2015 


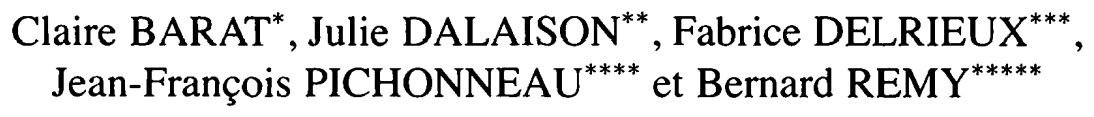

\section{PROSPECTION ET INVENTAIRE ARCHEOLOGIQUES DANS LA VALLEE DU GÖKIRMAK. PREMIER RAPPORT PRELIMINAIRE : REGION DE TAŞKÖPRÜ}

Dès l'année 2007, une première collaboration franco-allemande a permis de mettre en place et de définir un programme de prospection pluriannuel dans la région de Taşköprïi ${ }^{1}$. Un inventaire archéologique des vestiges architecturaux dispersés dans les villages et quartiers environnant le site de Pompeiopolis à Taşköprü a été entrepris à l'été 2008 par une équipe pluridisciplinaire 2 .

Sans être totalement ignorée par la recherche scientifique, la région a été peu étudiée, malgré son fort potentiel archéologique. Ainsi, dès le début du XIX $^{\mathrm{e}}$ s., Pascal Fourcade, consul général de France à Sinope ${ }^{3}$, avait parcouru la ville de Taşköprü et sa campagne environnante, où il avait repéré plusieurs inscriptions - qui lui avaient permis d'identifier avec certitude Taşköprü à Pompeiopolis - et de très nombreux vestiges architecturaux et céramiques $^{4}$. Dans une publication de 1915, Richard Leonhard avait produit un travail fondateur sur la
Paphlagonie ${ }^{5}$ et apporté une étude sur les tombes rupestres de la vallée du Gökırmak. Hubertus Von Gall avait étudié en détail les tombes rupestres de Paphlagonie dans un ouvrage paru en $1966^{6}$. En 1977, Pauline Donceel-Voûte avait dirigé une mission dans la vallée du Gökırmak et avait mentionné rapidement la découverte de quelques sites (non décrits) ${ }^{7}$. Entre 1995 et 1997, Catherine Marro, Asl 1 Özdoğan, Aksel Tibet et Catherine Kuzucuoğlu avaient mené trois campagnes de prospection, surtout centrées sur la pré- et la protohistoire ${ }^{8}$.

Le but de cette première mission était de recenser les sites antiques ou au moins de repérer les vestiges gréco-romains, car Pompeiopolis a servi de carrière à toute la région après sa destruction systématique, peut-être par un chef de guerre arabe lors de l'invasion de l'Anatolie au VII ${ }^{\mathrm{e}}$ s. ${ }^{9}$, et des éléments d'architecture. Le recensement a donné lieu à une base de données ${ }^{10}$ dans le cadre de l'étu-

*) Université de Valenciennes et du Hainaut-Cambrésis.

**) Université de Lyon II.

***) Université de Savoie.

$* * * *$ ) SRA, DRAC Aquitaine

$* * * * *$ ) Université de Grenoble II.

1) Claire Barat avait effectué un premier travail de recherche sur la région de la mer Noire dans le cadre de sa thèse de doctorat sur "Sinope dans son environnement pontique", soutenue en décembre 2006 à I'Université de Bordeaux 3. La collaboration avec Lâtife Summerer, Professeur à l'Université de Munich, spécialiste de la région du Pont et directrice des fouilles de Pompeiopolis semblait évidente pour une meilleure synergie.

2) Grâce à l'autorisation accordée par la Direction Générale des Antiquités Turques et à un financement de l'université de Lyon II et de la Maison de l'Orient Méditerranéen (cluster 13), la mission de prospection a pu avoir lieu du 18 au 28 août 2008 . A l'équipe française se sont joints ponctuellement un certain nombre de membres de l'équipe allemande des fouilles de Pompeiopolis : I âtife Summerer, Professeur en archéologie classique à l'Université Ludwigmaximilan de Munich et directrice des fouilles de Pompeiopolis ; Christian Marek, Professeur d'histoire ancienne à l'Université de Zurich ; Dr. Alexander Von Kienlin, architecte-ingénieur à l'Université Technique de Munich ; Dr. Jörg Faßbinder, géophysicien au Bayerische Landesamt für Denkmalpfledge. Nous avons eu la chance de bénéficier du concours scientifique et administratif et de la gentillesse de Funda Ünal, commissaire de fouilles et représentante de la Direction Générale des Antiquités Turques.

3) Barat $2003: 163-175$.

4) Fourcade $1811: 30-58$.

5) Leonhard 1915.

6) Von Gall 1966.

7) Donceel-Voûte $1979:$ 196-197.

8) Marro, Özdoğan, Tibet $1996: 273-290$; Marro, Özdoğan, Tibet, Kuzucuoğlu 1997 : 275-306 ; Marro, Özdoğan, Tibet, Kuzucuoğlu $1998: 317-335$.

9) Mention d'une tradition orale par Pascal Fourcade au début du XIX ${ }^{\mathrm{e}} \mathrm{s}$. : le sultan qui s'était emparé de la ville , irrité par la résistance des habitants, s'était juré de détruire la ville pierre par pierre : Fourcade 1811:37.

10) Cette base de données est mise à la disposition du Musée archéologique de Kastamonu et du service municipal de Taşköprü en vue de la conservation et de la présentation de ces éléments architecturaux. 


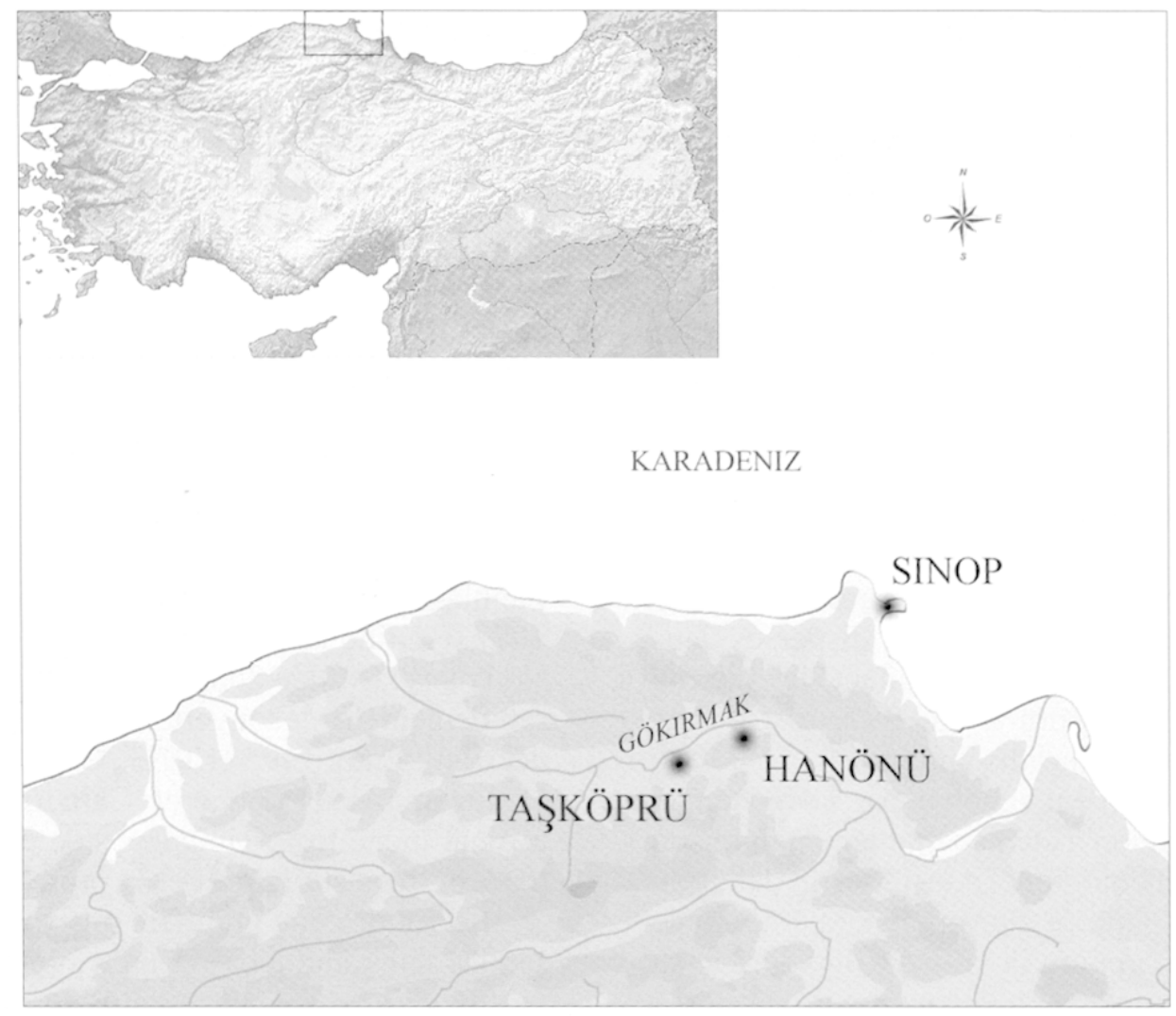

Fig. 1 : Carte de localisation de la région de Taşköprü.

de du territoire de Pompeiopolis. Pour ce premier contact, nous avons décidé de prospecter dans la ville de Taşköprü, ce qui n'avait pas été fait depuis P. Fourcade, et seulement dans les villages environnants, où des "antiquités" nous avaient été signalées par des habitants de Taşköprü ou par des ouvriers turcs de la fouille. Un très grand nombre de blocs taillés ont été repérés, mais nous avons pris le parti de ne retenir que ceux qui étaient ornés ou qui présentaient au moins des moulures. Ceuxci ont fait l'objet d'une fiche descriptive et d'un recensement cartographique sur le parcellaire.

Nous avons ainsi retenu cent trente-cinq éléments d'architecture pour la commune de Taşköprü et quarante-sept pour les zones rurales que nous avons prospectées (Fig. 2). Sur la rive gauche du Gökırmak, nous avons exploré la zone comprise entre l'Aygır Dağı et le Çangal Dağı (villages de Bademci et de Donalar), puis la zone au Nord de Taşköprü, comprise entre le Çangal Dağı et un petit affluent asséché du Gökrrmak (villages de Beyköy et Yeniköy). Sur la rive droite du Gökırmak, nous avons prospecté une partie de la vallée au Sud de
Taşköprü, le long de la route de Çiftlikköy (villages de Kızlkise et Kise Köyü), ainsi qu'une partie de la zone montagneuse de l'Elek Dağı (villages de Bodur et de Meye). Toujours sur la rive gauche du Gökırmak, légèrement au Nord-est de Taşköprü, nous avons prospecté les communes de Kızkalesi et Çarıklı. Enfin, nous nous sommes rendus dans la commune de Hanönü, où un caravansérail avec des éléments d'architecture antique avait été signalé par Pascal Fourcade lors de son voyage en $1806^{11}$.

\section{TAŞKÖPRÜ (ZONES 1 A 15)}

La ville moderne de Taşköprü est située sur la rive droite du Gökırmak (antique Amnias) à moins d'un kilomètre du site de Pompeiopolis qui occupait une colline (Zımbıllı Tepe) de la rive gauche. La rivière est franchie par un pont à six arches en pierre qui a donné son nom à la ville. Ce pont avait été décrit par Pascal Fourcade lors de son passage à Taşköprü. Ce dernier avait mentionné des blocs en remploi dans les murs des maisons et d'un collège, dans les cimetières et les mosquées ${ }^{12}$. Vital 


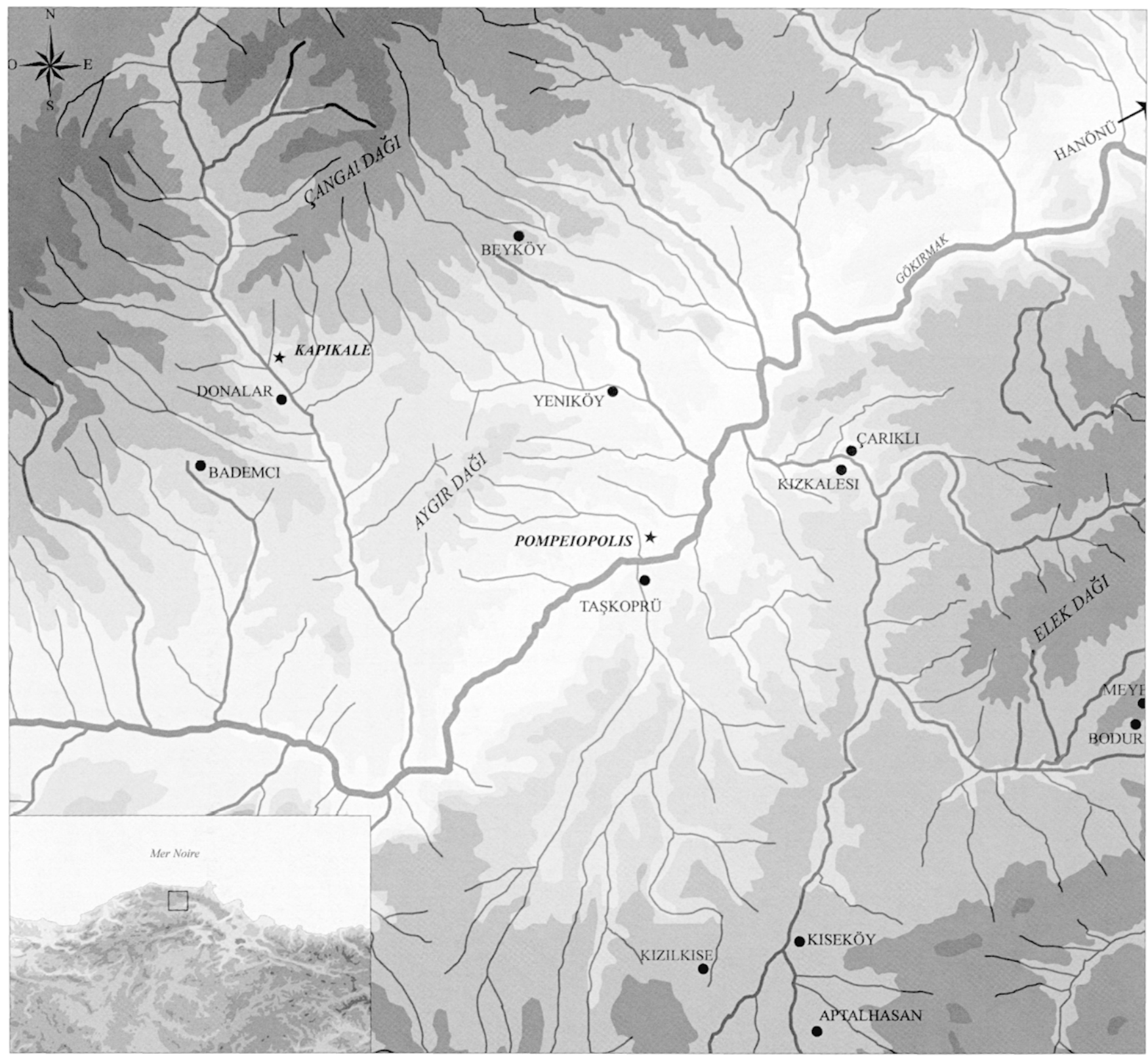

Fig. 2 : Carte de la zone prospectée.

Cuinet, lors de son tableau statistique de la Turquie d'Asie à la fin du XIX ${ }^{\mathrm{e}}$ s., mentionne également la présence d'un bâtiment carré en face de la grande mosquée sur la place principale. Il signale que les habitants appelaient ce bâtiment l'école et que son toit renversé était soutenu par dix colonnes de marbre d'ordres différents (six colonnes corinthiennes, deux colonnes doriques et deux colonnes toscanes). Vital Cuinet a également précisé que ces colonnes mesuraient cinq mètres et portaient quelques traces d'inscriptions devenues illisibles ${ }^{13}$.
Lors de notre prospection du centre-ville de Taşköprü, l'aspect général du centre-ville n'a pas été conforme à celui évoqué par nos deux sources $\mathrm{du} \mathrm{XIX}^{\mathrm{e}} \mathrm{s}$. en raison d'une modernisation des quartiers correspondants avec un aménagement de zones piétonnes et de larges rues. Les vestiges devaient se trouver en face de l'actuelle Taş Camii, près de la place principale et du jardin public. Certains vestiges antiques ont pu être mis en valeur dans des fontaines publiques transportés au dépôt situé au pied de la colline de Zımbilh. La majorité 


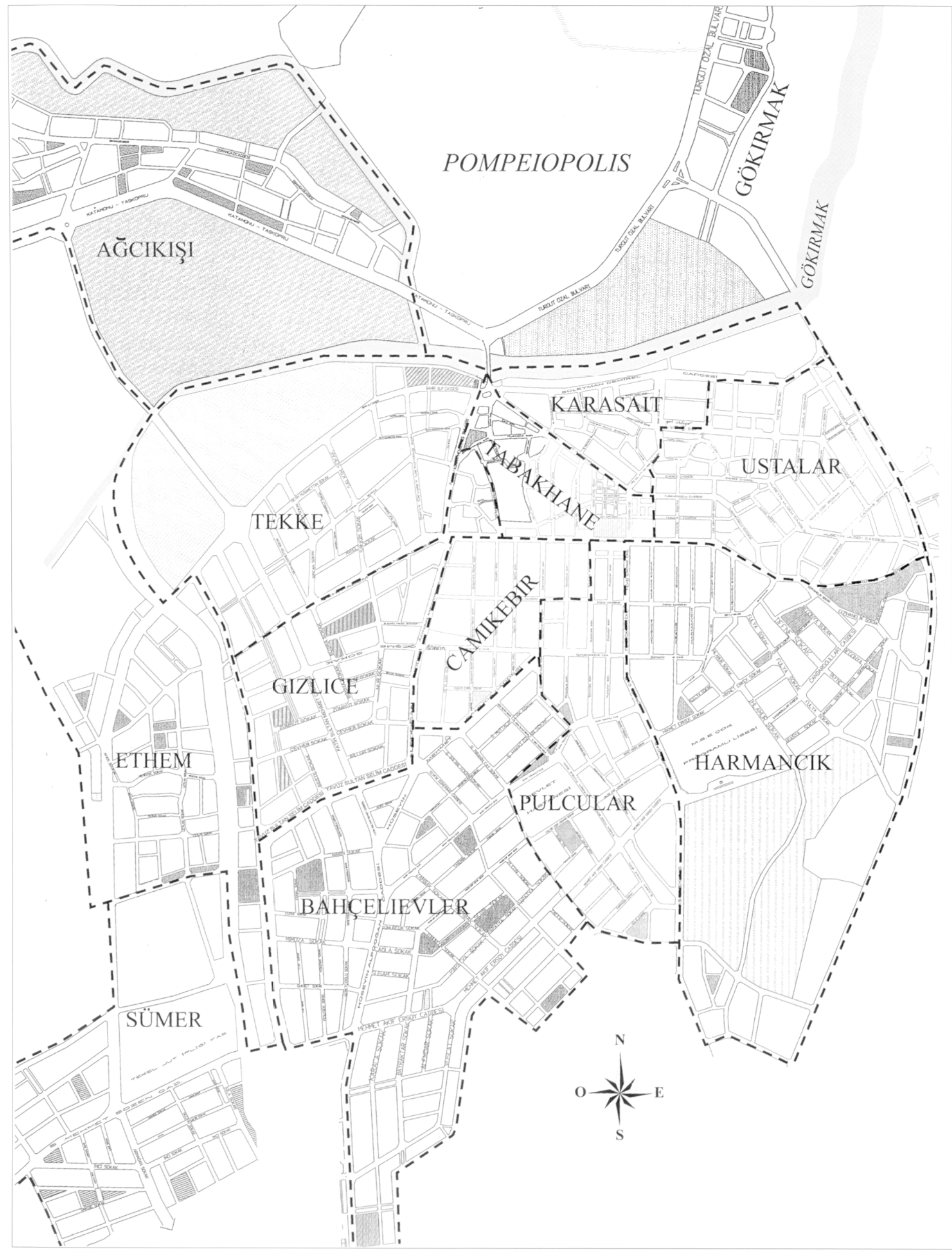

Fig. 3 : Plan de Taşköprü et division par quartiers. 
de nos trouvailles sont situées dans des quartiers anciens ou des villages qui ont été rattachés à la commune de Taşköprü, dans un habitat traditionnel.

Pour notre prospection, nous avons suivi la division municipale de Taşköprü (Fig. 3) en quatorze quartiers (Mahalle) ${ }^{14}$, en rajoutant un quinzième quartier pour le site de Pompeiopolis (Zımbıllı Tepe). Seuls huit quartiers ont livré un nombre non négligeable de vestiges très variés, soit en remploi dans une construction, soit posés sur le trottoir ou dans un jardin. Nous en avons recensé cent trente-cinq : quatre à Tabakhane (quartier 1), un à Karasait (quartier 2), quatre à Musalla (quartier 7), deux à Gizlice (quartier 8), vingt à Tekke (quartier 9), quatre à Bahçelievier (quartier 10), vingt-cinq à Ethem (quartier 11), deux à Sümer (quartier 12) et soixante et onze à Ağcıkışı (quartier 13).

Trois quartiers se détachent donc : Ağcıkışı, Tekke et Ethem, qui cumulent cent seize trouvailles sur les cent trente-cinq de la commune de Taşköprü.

Le quartier d'Ağcıkışı (quartier 13) se situe au Nord de la commune de Taşköprü, sur la rive gauche du Gökırmak, au-dessus de la route venant de Kastamonu. Ce quartier a la particularité d'être un ancien village rattaché à la commune, mais il a conservé un habitat traditionnel rural, un vieux cimetière et un grand terroir agricole. Ce quartier ne se situe pas sur la même rive du Gökırmak que les quartiers centraux, mais sur une colline à proximité de Zımbıllı Tepe. Une enquête auprès de la population a d'ailleurs montré que les habitants d'Ağcıkışı possèdent leurs terres agricoles sur Zimbillı Tepe et que de nombreux blocs d'architecture présents dans le quartier viennent de l'épierrement de leurs champs. Le nom même d'Ağcıkışı peut être significatif car, si on le décompose étymologiquement, on voit qu'il comprend le mot "kişi", qui signifie "personnes, gens" et "ağcı", qui est peut-être l'adjectif dérivé de "ağ", qui signifie "filet, rets". En rapprochant cette étymologie de la tradition de la destruction de la cité de Pompeiopolis rapportée par Pascal Fourcade, nous pouvons émettre l'hypothèse qu'une fois la cité détruite au VII ${ }^{\mathrm{e}}$ s., les survivants, prisonniers "pris dans les rets" ont pu être déplacés et réinstallés sur la colline la plus proche du site, en récupérant ce qui pouvait l'être pour construire un village.
La majorité des vestiges ont été découverts dans les rues principales d'Ağcıkışı (Fig. 4), au cœur de l'ancien village : Orhan Gazi Sokak, Osmangazi Caddesi, Genç Osman Sokak et Ertuğrul Gazi Sokak, dans la zone autour de la mosquée de quartier. La prospection du cimetière en contrebas de la Genç Osman Sokak et du jardin de la mosquée a également apporté son lot de découvertes, tant dans la partie moderne que dans la partie abandonnée.

Nous avons commencé notre prospection des quartiers de Taşköprü par le quartier d'Ağckıışı en raison de la présence dans le jardin de la mosquée d'une base quadrangulaire surmontée d'un globule posé sur une moulure (h. : $103 \mathrm{~cm} ; \mathrm{d} .: 143 \mathrm{~cm}$ ) et percé au sommet d'une cavité circulaire dans un carré creux (Fig. 5). Cette pièce était connue depuis la publication de $\mathrm{H}$. Von Gall sur les tombes rupestres en Paphlagonie ${ }^{15}$. Ce savant a étudié cette base et envisagé sa finalité : base de colonne d'une tombe rupestre ? Cippe décoratif sur le sommet d'une tombe monumentale ? Phallus monumental ? Il peut s'agir d'une base de colonne provenant d'un édifice dont le style nous renvoie à une tradition locale, antérieure ou contemporaine à l'époque romaine ${ }^{16}$. Sans pouvoir l'affirmer, malgré sa taille et son poids imposants, cette base a peut-être été transportée d'assez loin ou bien extraite in situ afin d'orner le jardin de la mosquée d'A ğcıkışı lors de sa construction. La présence de la cupule située sur le dessus du bloc permet peut-être de penser que cette base a été réutilisée comme un autel dès l'Antiquité tardive.

Le reste des vestiges est composé de huit bases et de huit fûts de colonnes, de quatre architraves, de deux chapiteaux, de trois corniches, de deux pilastres d'angle, de trois fragments de plafonds à caissons, de deux chambranles, de deux seuils, d'un fragment de balustrade, d'une console, de deux soubassements, de deux dalles, et de vingtneuf blocs moulurés de tailles diverses.

La majorité de ces vestiges sont en marbre blanc et proviennent des monuments publics de Pompeiopolis. Les blocs recensés sont tous attribuables au Haut et au Bas Empire. Leur présence est liée à la transformation du site antique en espace agricole et au fait que les habitants d'Ağcıkışı sont propriétaires des terres agricoles de Zimbillı Tepe. Cela explique aussi que certains

14) Nous tenons ici à remercier M. Mustafa Günay, maire de Taşköprü, qui a gracieusement mis à notre disposition toutes les ressources des services techniques.

15) Von Gall $1966: 113-116 ;$ pl. 16. 1. 2

16) La présence du tore nous rattache à la période romaine. 


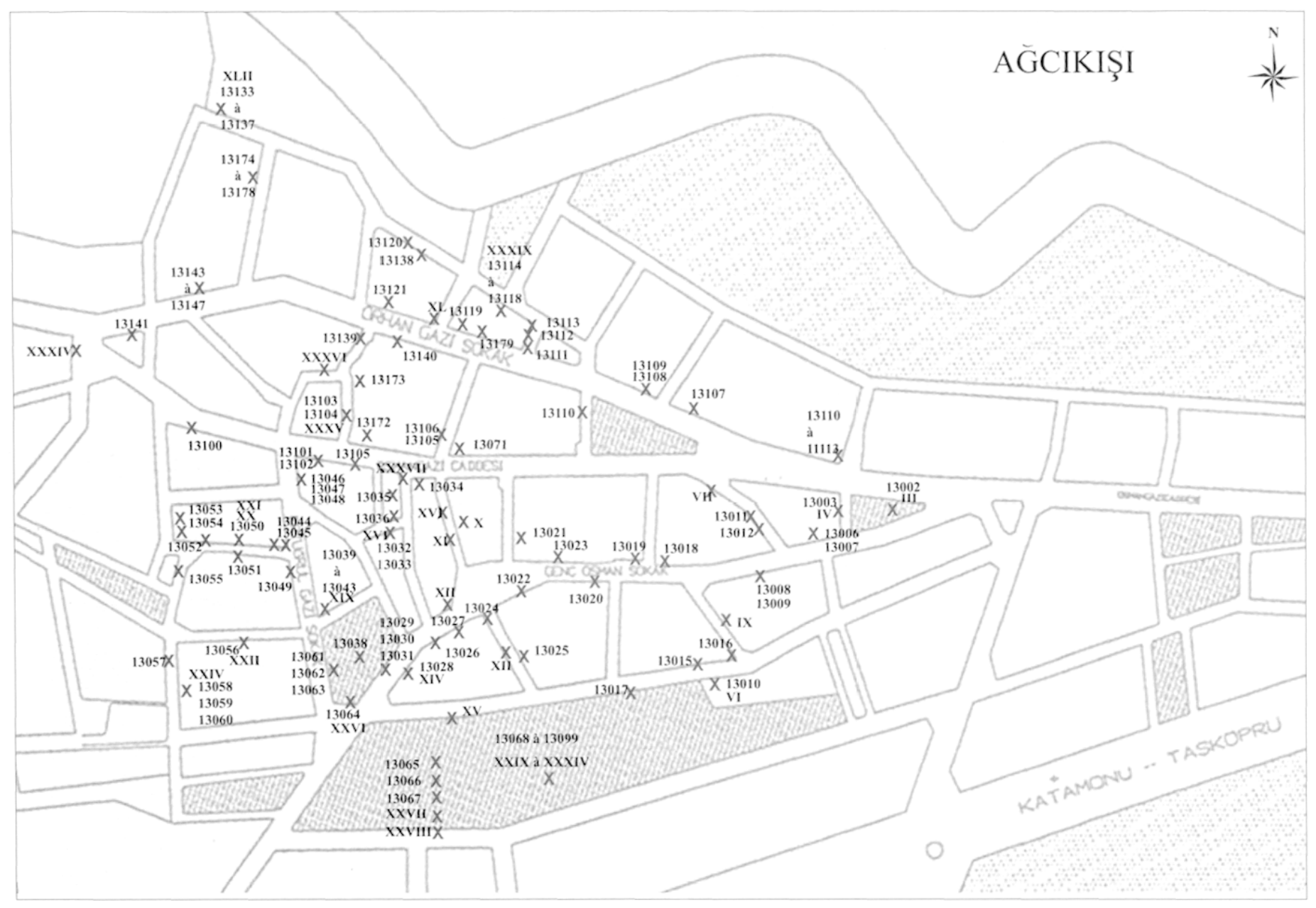

Fig. 4 : Plan du quartier d'Ağcıkışı et localisation des trouvailles.

blocs ont été partiellement évidés pour être remployés comme mortiers. Seuls quatorze éléments sont en remploi dans les constructions, ce qui signifie que cinquante-sept éléments se trouvent dans les rues ou dans les champs et risquent de disparaître. Les éléments les plus menacés sont ceux qui se situent dans les champs d'ail et de betteraves entre la route de Kastamonu, Osmangazi Caddesi et la colline de Zımbillı 'Tepe. C'est dans cette zone que des fragments de tubuli et de dalle de suspensura ont été découverts. Ils sont peut-être à mettre en relation avec l'établissement thermal (en cours de fouille) situé sur le flanc ouest de la colline de Zımbıllı Tepe, donc mitoyen du village d'Ağcıkışı.

Sur la rive droite du Gökırmak, de l'autre côté du pont de pierre se situe Tekke (quartier 9), le quartier le plus ancien de Taşköprü organisé autour d'une mosquée d'époque seldljoukide ${ }^{17}$. Vingt éléments d'architecture ont été recensés, en particulier autour de la mosquée de Tekke et dans les rues au tracé le plus ancien (Bahri Alp Caddesi, Katipoğlu Sokak et Şeyh Hüsamettin Sokak). La moitié des éléments est en remploi dans les bâtiments (notamment dans les murs de la mosquée). Un inventaire partiel a déjà été mené par la municipalité de Taşköprü et a pris en compte une bonne partie des vestiges se trouvant dans les rues ou les maisons particulières. La plupart des éléments sont en marbre blanc, de la période du Bas Empire et de l'époque paléochrétienne, moments où la ville a pu se développer sur la rive droite de l'Amnias. Nous avons recensé quatre bases et trois fûts de colonnes, trois fragments d'architrave, deux fragments de frise, un chapiteau ionique (Fig. 6), une cuve de sarcophage d'enfant avec des têtes de béliers, des bucranes et des guirlandes (Fig. 7). Enfin, sept blocs moulurés ont aussi été inclus dans notre inventaire.

17) Pompeiopolis fut en effet prise par l'émir seldjouk Hiisamettin Çoban en 1211. 


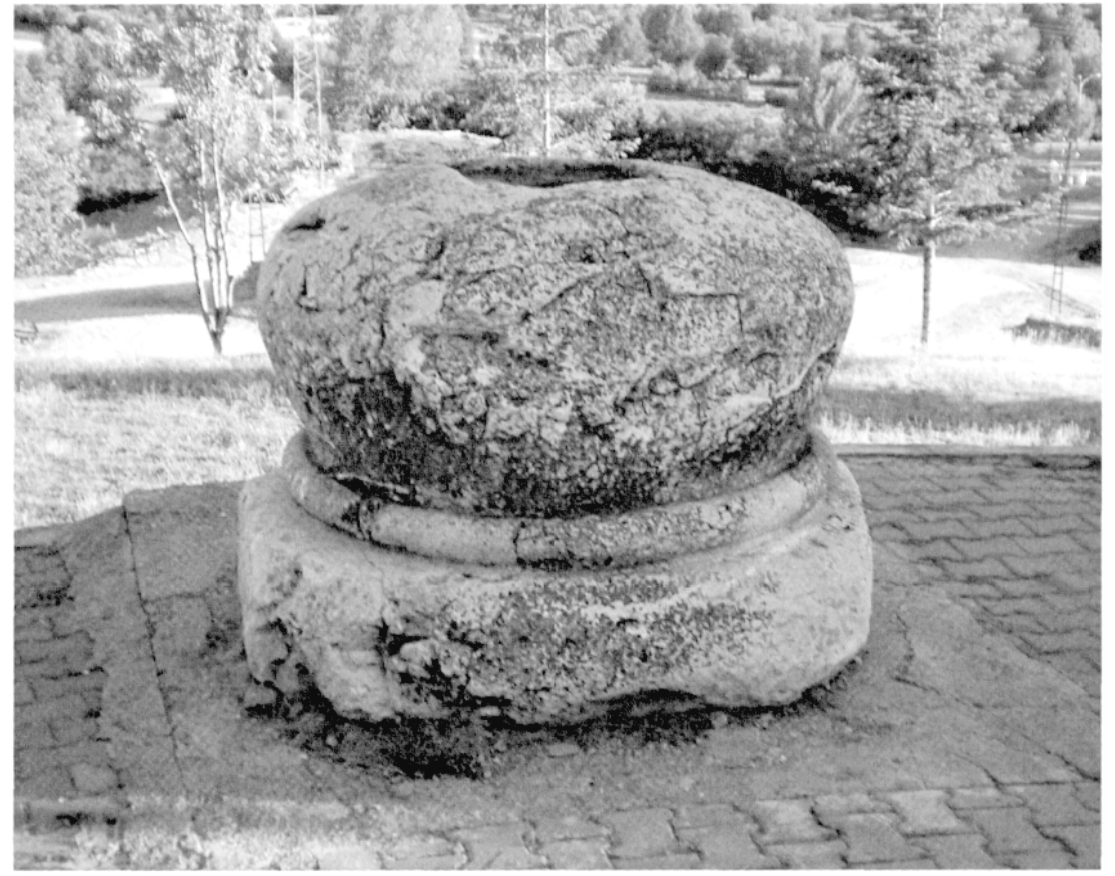

Fig. 5 : Taşköprü, quartier d'Ağcıkışı, base monumentale.

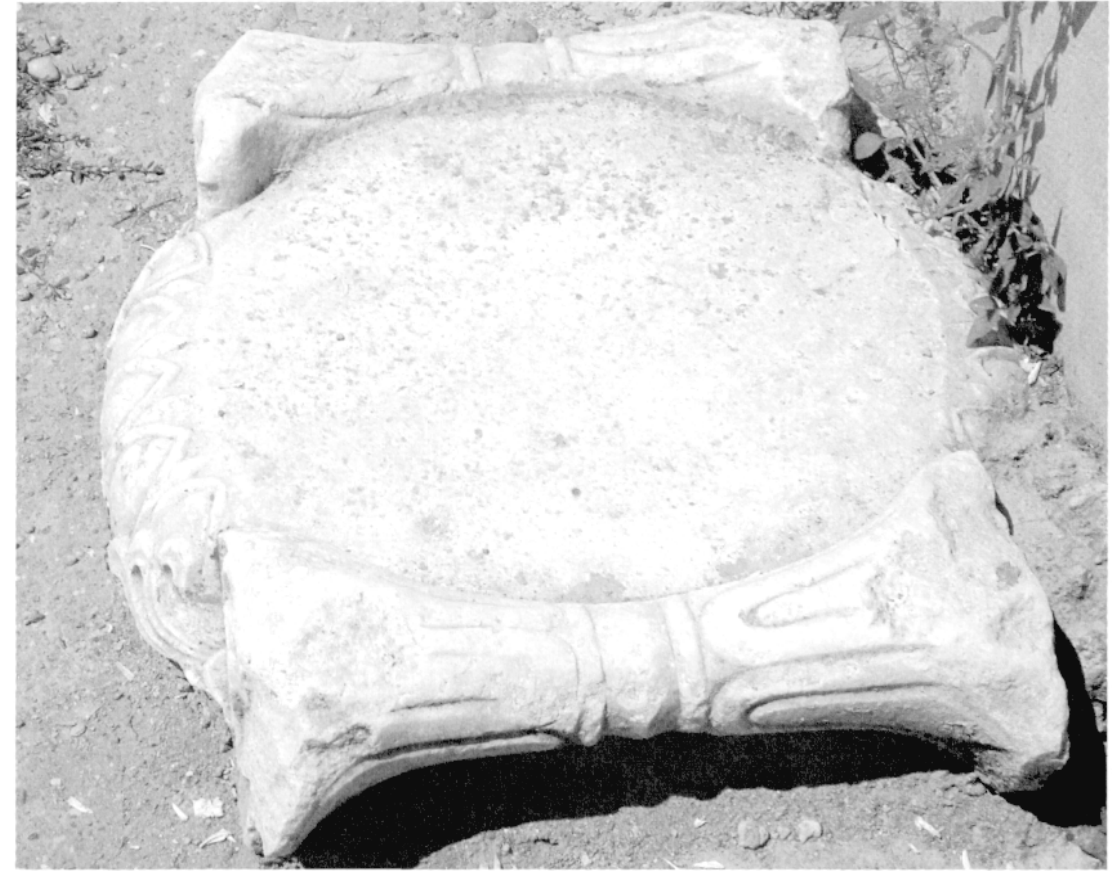

Fig. 6 : Taşköprü, quartier de Tekke, chapiteau ionique.

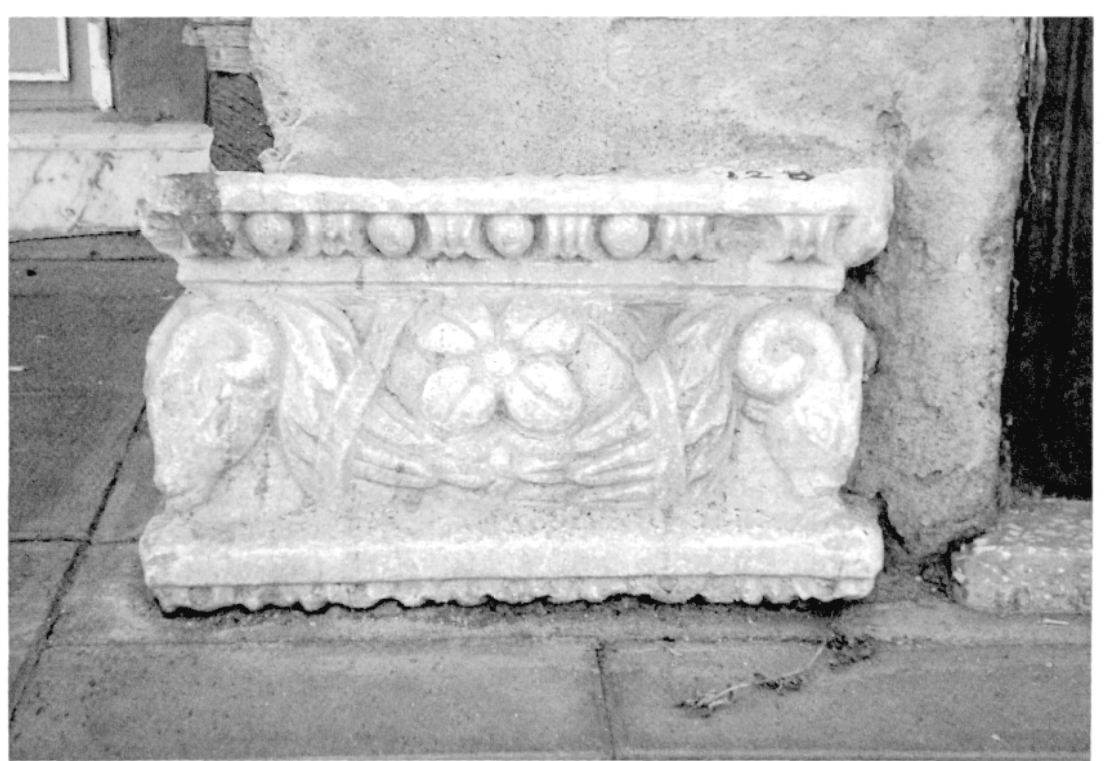

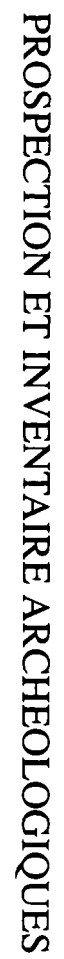

Fig. 7 : Taşköprü, quartier de Tekke, cuve de sarcophage d'enfant. 
Ethem (quartier 11) est le quartier mitoyen de Tekke au Sud-ouest et a fourni vingt-cinq éléments d'architecture. Tout comme Ağcıkışı, c'est un ancien village rattaché à Taşköprü et qui a gardé de nombreuses maisons traditionnelles où de nombreux blocs ont été utilisés en remploi. Ce quartier est séparé du reste de la commune de Taşköprü par le lit d'un affluent (aujourd'hui asséché) du Gökırmak. Comme à Ağcıkışı et à Tekke, ce sont dans les rues au tracé ancien que les blocs ont été en majorité découverts : Nilüfer Sokak, de part et d'autre du cours d'eau asséché, Menekşe Sokak, Nergis Sokak et Leylak Sokak. Nous avons découvert quatre fragments de frise (Fig. 9), deux fragments d'architrave (Fig. 8), trois fûts et deux bases de colonnes, un morceau de corniche, un morceau de linteau, une meule, onze blocs et une inscription (Fig. 10). Celle-ci se trouve sur un bloc de marbre blanc fragmenté (L. : $29 ; 1 .: 40 ;$ h. : 41). Le champ épigraphique est de $15 \times 39 \mathrm{~cm}$. Il s'agit d'une

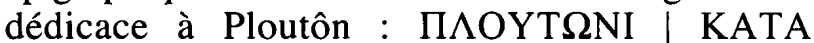

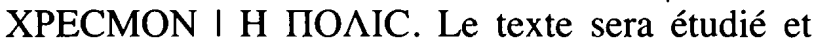
publié par Chr. Marek, professeur d'histoire ancienne à l'université de Zurich.

En dehors des trois quartiers d'Ağcıkışı, Tekke et Ethem, les autres quartiers de Taşköprü n'ont livré que dix-neuf éléments disparates : trois blocs, un fragment d'architrave, un morceau de plafond à caisson, un fragment de corniche, un fragment de cuve de sarcophage orné de guirlandes, de bucranes, de fleurons, de têtes et de grappes de raisin, un chapiteau corinthien, une base de statue, un seuil et deux fûts de colonnes. Deux inscriptions ont été découvertes : l'une, très fragmentaire, provient du quartier central de Tabakhane (quartier 1) et se compose de trois lettres de $12 \mathrm{~cm}$ de hauteur : OI $\Sigma$. L'autre inscription a été découverte à Bahçelievler (quartier 10) et comprend quatre lignes incomplètes d'une inscription en vers (Fig. 11). Cette inscription sera également étudiée et publiée par Chr. Marek. Signalons enfin deux colonnes à Musalla (quartier 7), utilisées en remploi dans une fontaine et provenant peut-être de la destruction du bâtiment à colonnes signalé en centre-ville par Vital Cuinet à la fin du XIX ${ }^{\mathrm{e}} \mathrm{s}$.

Comme nous venons de le voir, ces vestiges sont très souvent en marbre blanc et leurs décors sont soignés, ce qui atteste la qualité des monuments de Pompeiopolis, car il est certain que tous les vestiges retrouvés à Taşköprü proviennent de la ville antique, sauf peut-être ceux d'Ethem. L'homogénéité des trouvailles architecturales (architraves, frises très décorées en marbre blanc) dans ce quartier excentré pourraient indiquer l'existence d'un monument de belle taille et richement décoré. La découverte de la dédicace à Ploutôn à proximité immédiate (de l'autre côté du lit de l'ancienne rivière) inciterait à penser à un sanctuaire extra-urbain qu'il est impossible de localiser avec précision.

$\mathrm{Au}$ terme de ce premier inventaire, les principales découvertes se situant dans les quartiers d'Ağcıkışı, Tekke et Ethem, soit à l'Ouest du site antique de Pompeiopolis, il est possible de voir quelles furent les premières zones occupées après la destruction de la cité. Le quartier de Sümer (quartier 12), au Sud de Taşköprü et le long de la rivière asséchée qui passait à Ethem, mériterait une prospection plus systématique, en particulier son cimetière. Les actuels quartiers centraux n'ont que peu de chance de livrer d'autres vestiges car ils ont été rénovés récemment. Le quartier du Gökırmak (quartier 14), au pied du flanc est du site de Pompeiopolis et en bordure de la rivière sera aussi à prospecter car les zones agricoles renferment peut-être des vestiges antiques.

\section{LES VILLAGES ENVIRONNANTS}

\subsection{Rive gauche du Gökırmak, zone comprise entre l'Aygır Dağı et le Çangal Dağı : villages de Bademci (zone 17) et de Donalar (zone 19)}

Notre attention a été attirée dans cette zone par la présence de tombes rupestres déjà connues et étudiées, dans le cas de Donalar, par Richard Leonhard ${ }^{18}$ et Hubertus von Gall ${ }^{19}$.

A proximité du village de Bademci, nous avons observé une tombe rupestre (Fig. 12) déjà signalée par Pauline Donceel-Voûte lors de sa prospection de $1977^{20}$. Elle signalait aussi, à proximité du monument, la présence d'un aqueduc romain, qui n'a pas pu être repéré.

Dans le lit à sec d'un cours d'eau, grâce à une information du maire, nous avons découvert en deux endroits différents des pierres liées les unes aux autres par du mortier antique $(133 \times 63 \times 74 \mathrm{~cm}$; $750 \mathrm{~cm}$ ). Partiellement détruits, ces vestiges sont difficiles à identifier (murs ?). 


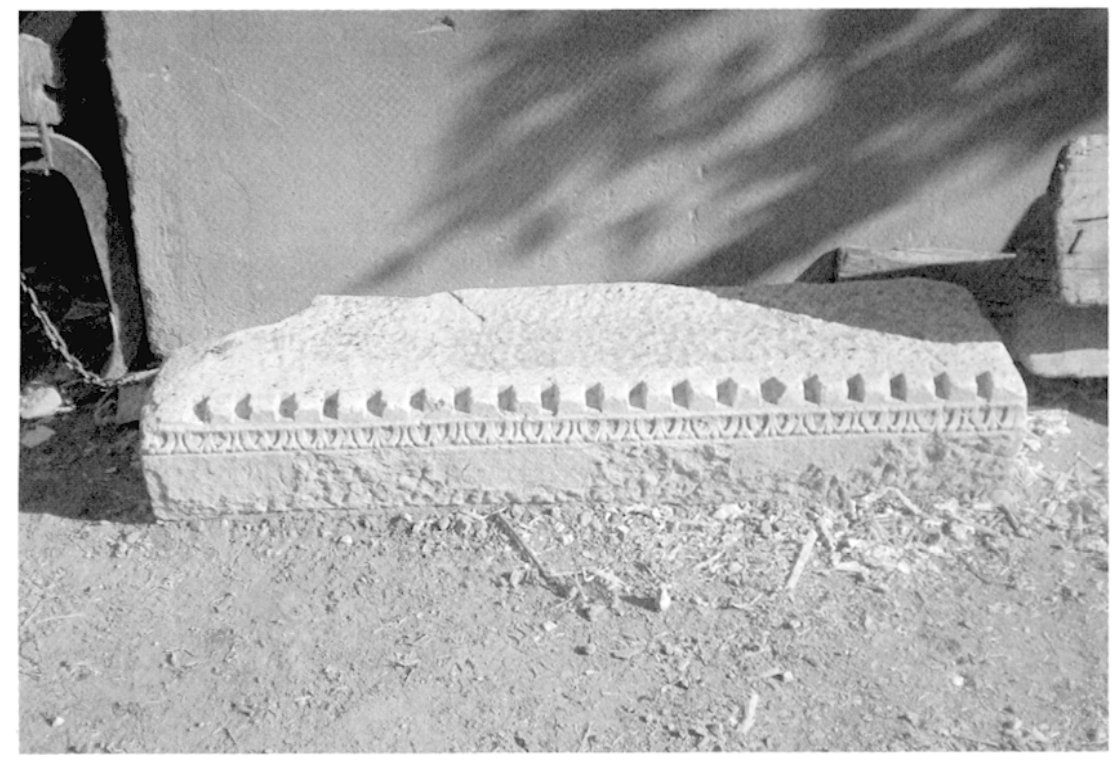

Fig. 8 : Taşköprü, quartier d'Ethem, fragment d'architrave.

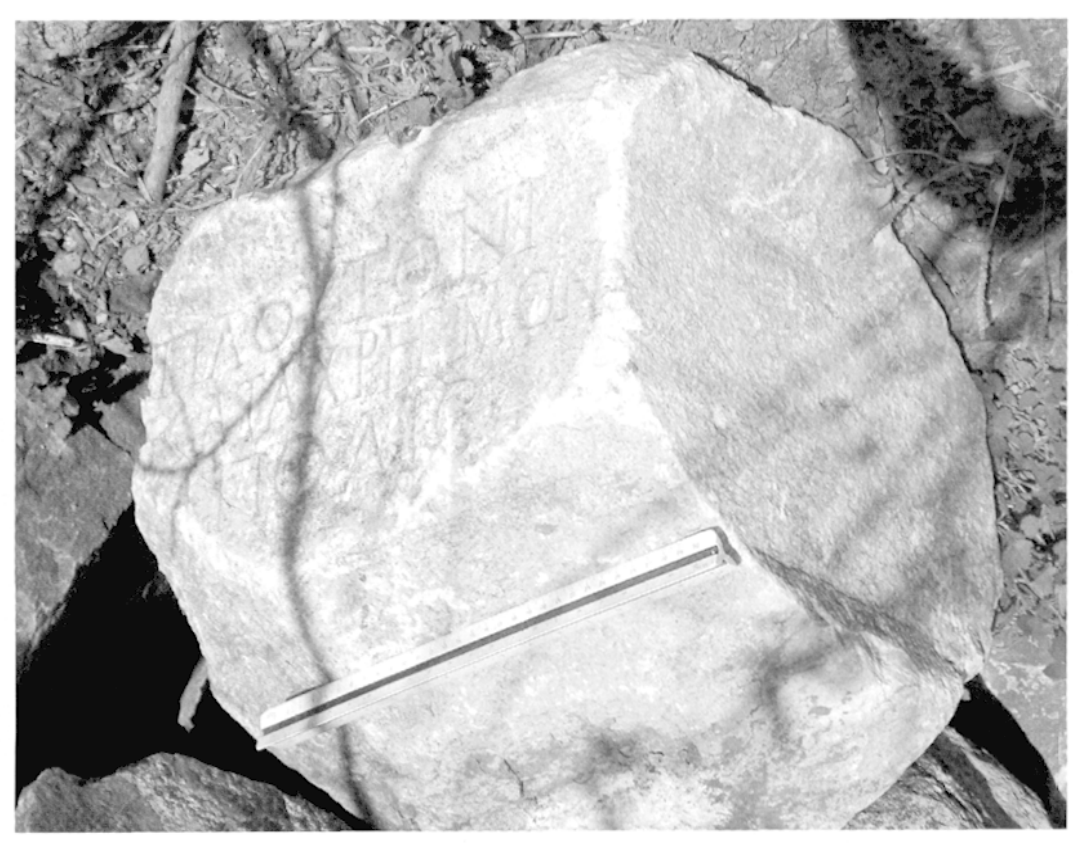

Fig. 10 : Taşköprü, quartier d'Ethem, dédicace à Ploutôn.

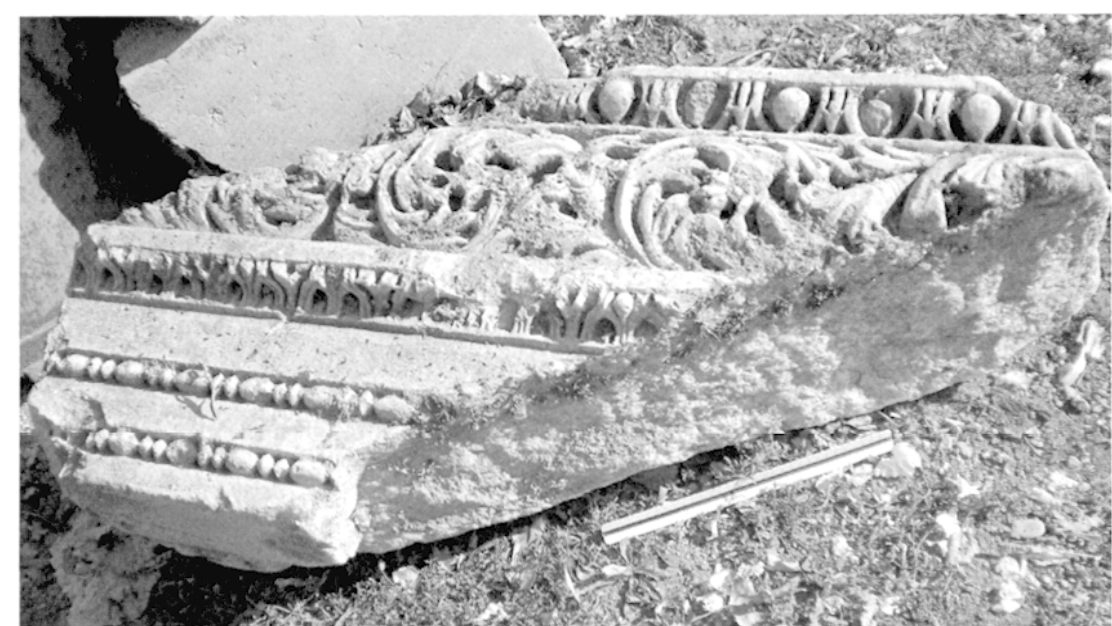

Fig. 9 : Taşköprü, quartier d'Ethem, fragment de frise.

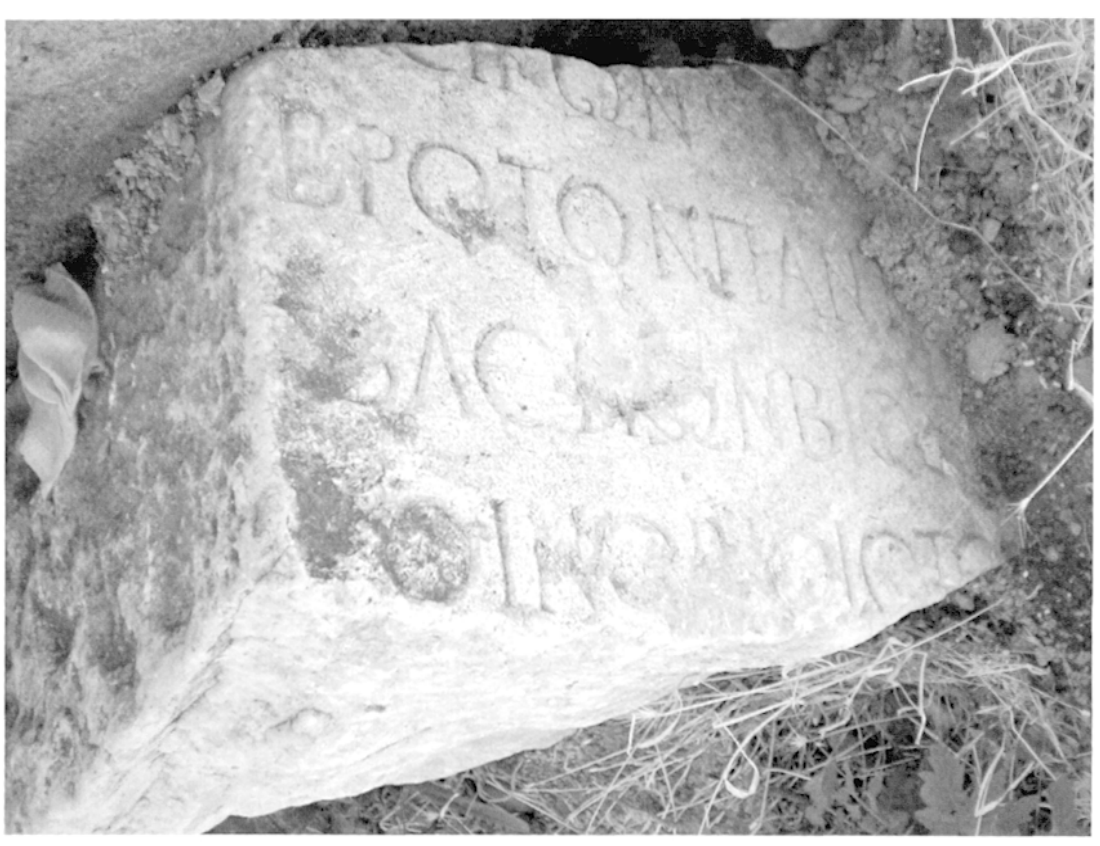

Fig. 11 : Taşköprü, quartier de Bahçelievler, inscription versifiée.

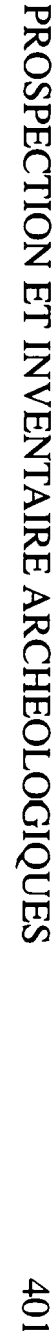


Dans le village même, nous avons seulement trouvé une colonne de marbre blanc, retaillée et évidée pour servir de mortier, et une colonne de calcaire à grain fin brisée en deux et évidée.

A Donalar, une tombe rupestre spectaculaire (Fig. 13) est connue et étudiée dès 1915, mais sur le même versant, nous avons repéré d'autres vestiges non publiés, ni même signalés. Sur le versant de la tombe apparaît un tunnel à escaliers (Fig. 14) taillé dans le rocher (profondeur actuelle : $47 \mathrm{~m}$, mais le tunnel est bouché au fond ; h. : $24 \mathrm{~m}$ ), avec l'empreinte de la porte, plusieurs entailles indéterminées dans la roche, un lit d'attente pour les blocs d'un mur, un escalier (Fig. 15). A mi-pente, une meule en ophite, cassée en deux $(47 \times 27 \times 17 \mathrm{~cm})$ est abandonnée sur le sol. On trouve aussi au pied du site des fragments de céramique et de tegulae. Sur le versant opposé est à signaler un second tunnel à escaliers (L. : $6,8 \mathrm{~m} ; 1 .: 3,8 \mathrm{~m}$ ), dont le plafond s'est en partie effondré.

Ce site devait être à l'évidence le siège d'un potentat local qui contrôlait toute la plaine agricole au Sud, au pied du site. L'endroit offrait ainsi un lieu d'habitation pour le dynaste, des murs de défense, des tours et des réserves d'eau (auxquelles on accédait par des escaliers taillés dans le rocher). L'érosion intense a entrainé la destruction générale de tous les vestiges en élévation. Seules sont restées les parties taillées dans la roche. Ainsi, on peut voir des replats, des escaliers, des trous de boulins et des lits d'attente taillés dans la roche. Le site sur piton offre une situation quasi imprenable et domine la vallée du Gökırmak jusqu'aux crêtes de l'autre rive.

Sur un autre versant, de l'autre côté de la piste qui conduit au barrage, nous avons découvert un tunnel à escaliers, des murs en gros blocs de calcaire et un escalier imposant dont les marches font plus de cinquante centimètres de haut (Fig. 16). Cet escalier permet d'accéder à une plate-forme sur laquelle pouvait être édifiée une tour ou un espace de surveillance. Situé de l'autre côté du cours d'eau, ce site complète le système défensif de Donalar et verrouille la vallée.

Les sites de Bademci et Donalar offrent des similitudes d'occupation. Ce sont des sites à flanc de coteau, orientés vers le Sud et la vallée du Gökırmak. Pauline Donceel-Voûte avait identifié de la céramique hellénistico-romaine sur les deux pentes au pied de Donalar et des tessons hellénisti- co-romains et byzantins dans trois zones au pied du monument rupestre de Bademci ${ }^{21}$. Seule une prospection céramologique systématique permettrait de dire si ces sites ont été occupés à des époques antérieures.

\subsection{Zone au Nord de Taşköprü, comprise entre le Çangal Dağı et un petit affluent asséché du Gökırmak : villages de Beyköy (zone 18) et Yeniköy (zone 16).}

Le village de Beyköy se situe sur le même axe nord-est / sud-ouest que les villages de Bademci et Donalar, entre Çangal Dağı et Aygır Dağı. Dans ce village, prospecté par C. Marro, A. Özdoğan et A. Tibet en $1995^{22}$, nous avons retrouvé les vestiges assez bien conservés d'un bâtiment antique $(4 \mathrm{x}$ $4,20 \mathrm{~m}$ ), construit en moellons calcaires assez irréguliers et liés par du mortier (Fig. 17). Un mur avec une voûte est encore debout sur une hauteur de $1,80 \mathrm{~m}$. Faut-il envisager qu'il puisse s'agir des thermes d'une uilla ? Il semblerait que ce bâtiment (ou la maison de maître) ait servi de carrière, notamment pour construire et orner les deux mosquées. Dans les murs sont concentrés un bon nombre de pièces d'architecture en marbre blanc: blocs monumentaux (un orné d'un fleuron, d'une grappe de raisins, d'un quadrupède tourné à gauche et d'une feuille (Fig. 18) ; un autre décoré d'un fleuron inscrit dans une couronne de feuillage), et une corniche fragmentaire à denticules.

Dans le jardin des mosquées sont rassemblés des éléments d'architecture en marbre blanc : trois colonnes fragmentaires à astragale dont une avec des cannelures à arêtes plates (h.: $101 \mathrm{~cm} ; \mathrm{d}$. : 55 $\mathrm{cm}$ ), deux lisses (h. : $104 \mathrm{~cm} ;$ d. $29 \mathrm{~cm} ;$ h. : 50 $\mathrm{cm} ; \mathrm{d} .: 33 \mathrm{~cm})$ et un chapiteau corinthien (h. : 36,5 $\mathrm{cm} ; \mathrm{d} .: 31 \mathrm{~cm})$.

Entre Beyköy et la route principale permettant de revenir à Taşköprü par le Nord, le long d'un petit affluent du Gökırmak, P. Donceel-Voûte avait signalé l'existence d'un site (sans le décrire) à Yeniköy Kuyucak ${ }^{23}$. Dans le village, nous avons inventorié un fût de colonne en calcaire à grain fin, une base de statue $(27 \times 46,5 \times 85 \mathrm{~cm})$ en marbre et surmontée d'une corniche avec un trou de fixation pour la statue, un seuil $(198 \times 61 \times 34 \mathrm{~cm})$, un chambranle gauche en marbre blanc $(220 \times 50 \times 22 \mathrm{~cm})$, un fragment de sarcophage avec des grappes de raisin, 


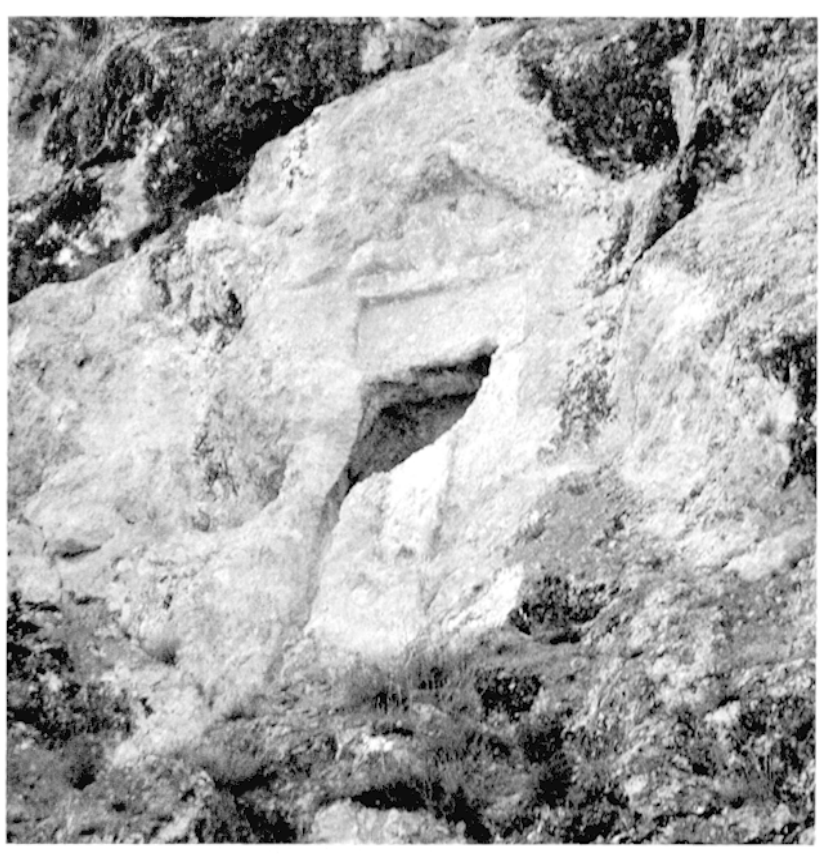

Fig. 12 : Bademci, tombe rupestre.

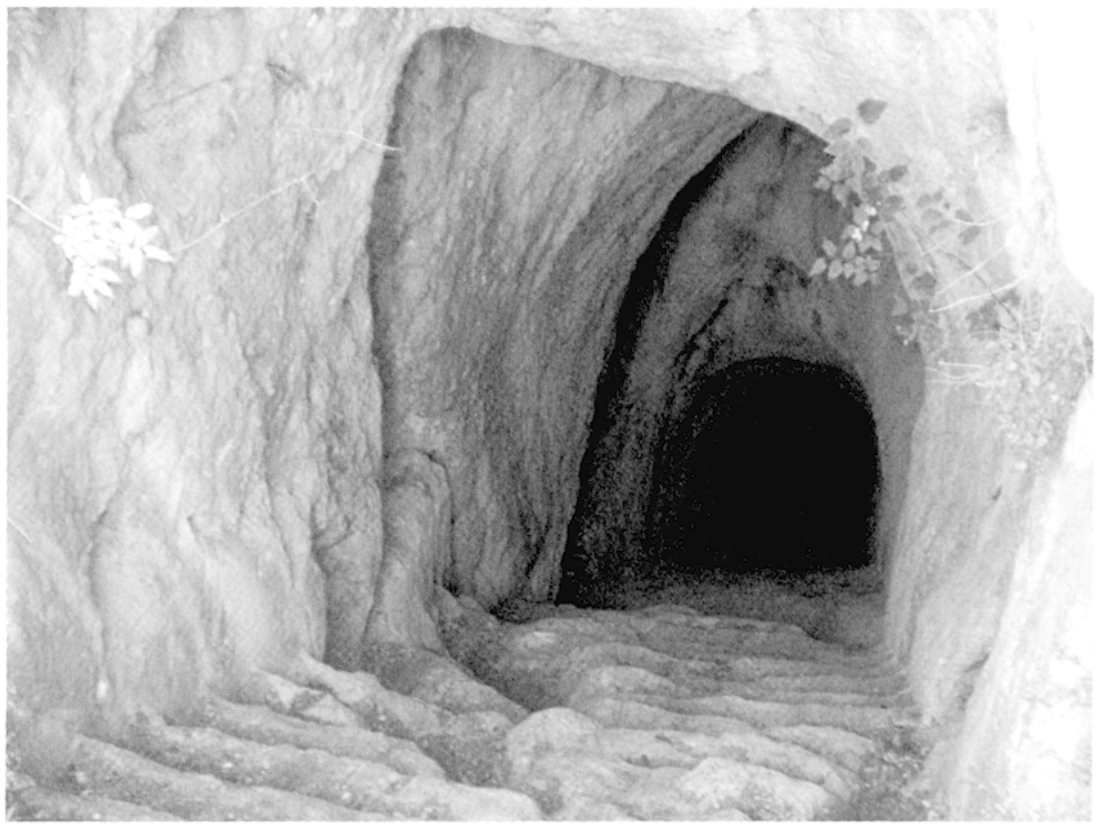

Fig. 14 : Donalar, tunnel à escaliers.

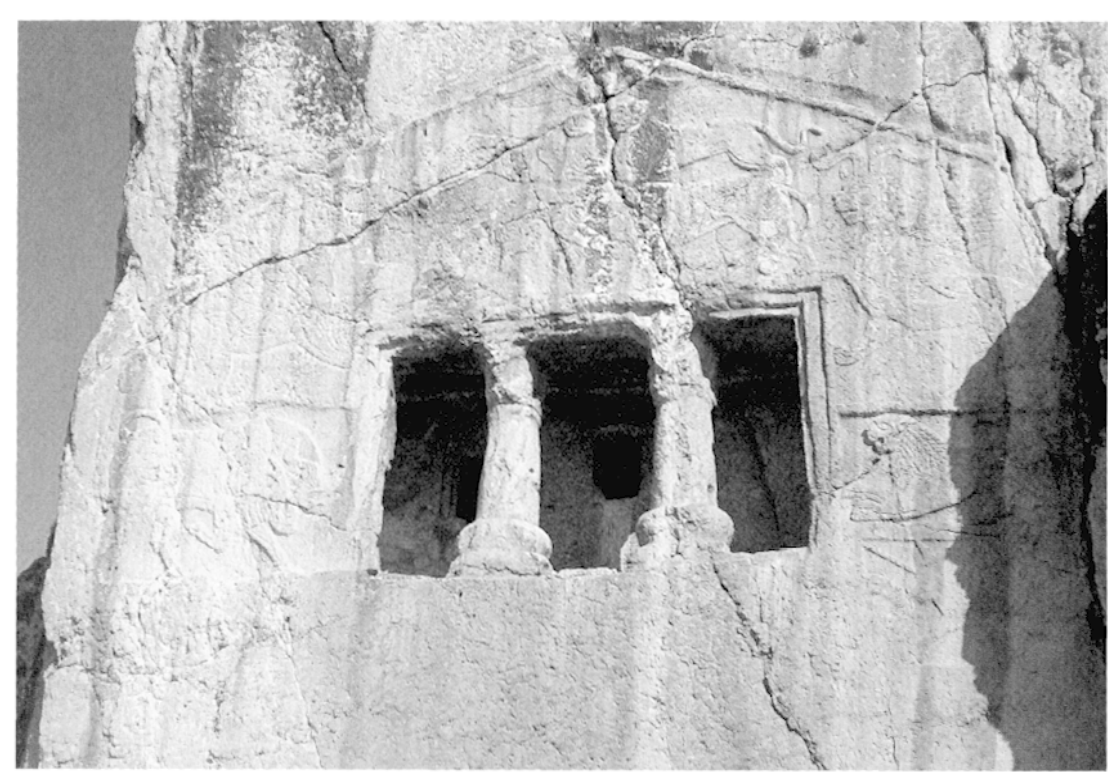

Fig. 13 : Donalar, tombe rupestre.

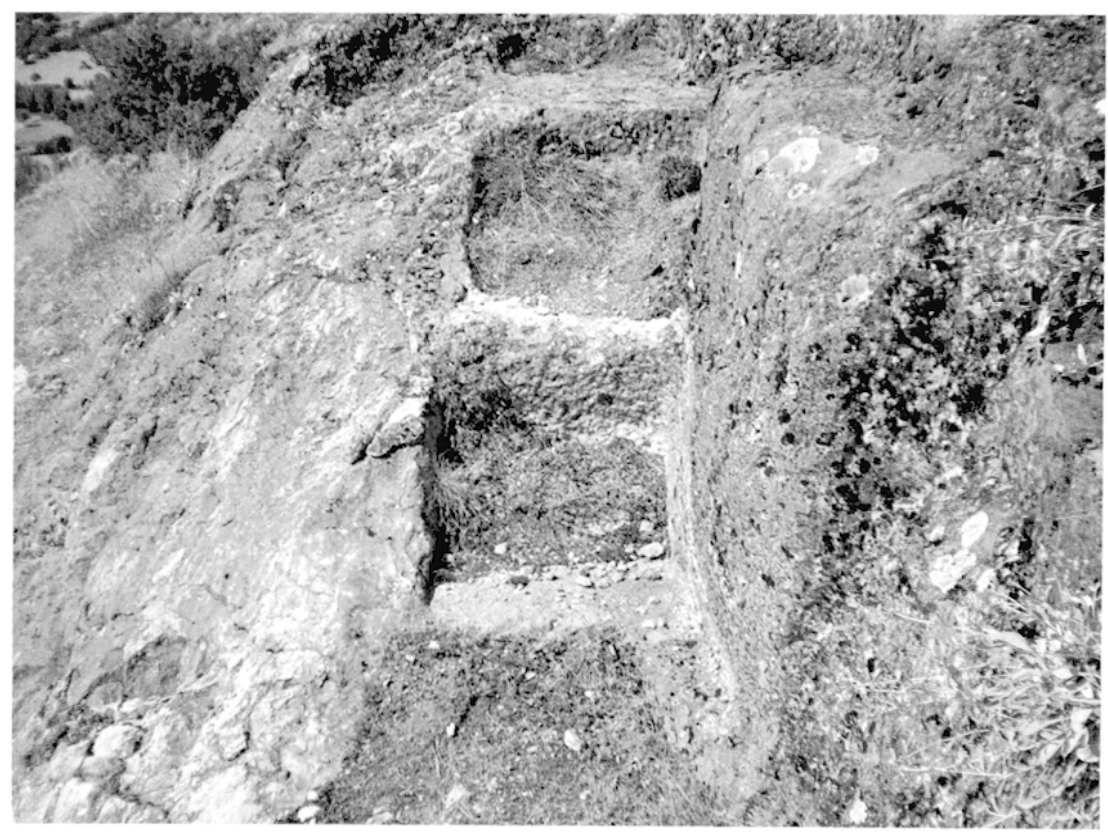

常

Fig. 15 : Donalar, escaliers. 


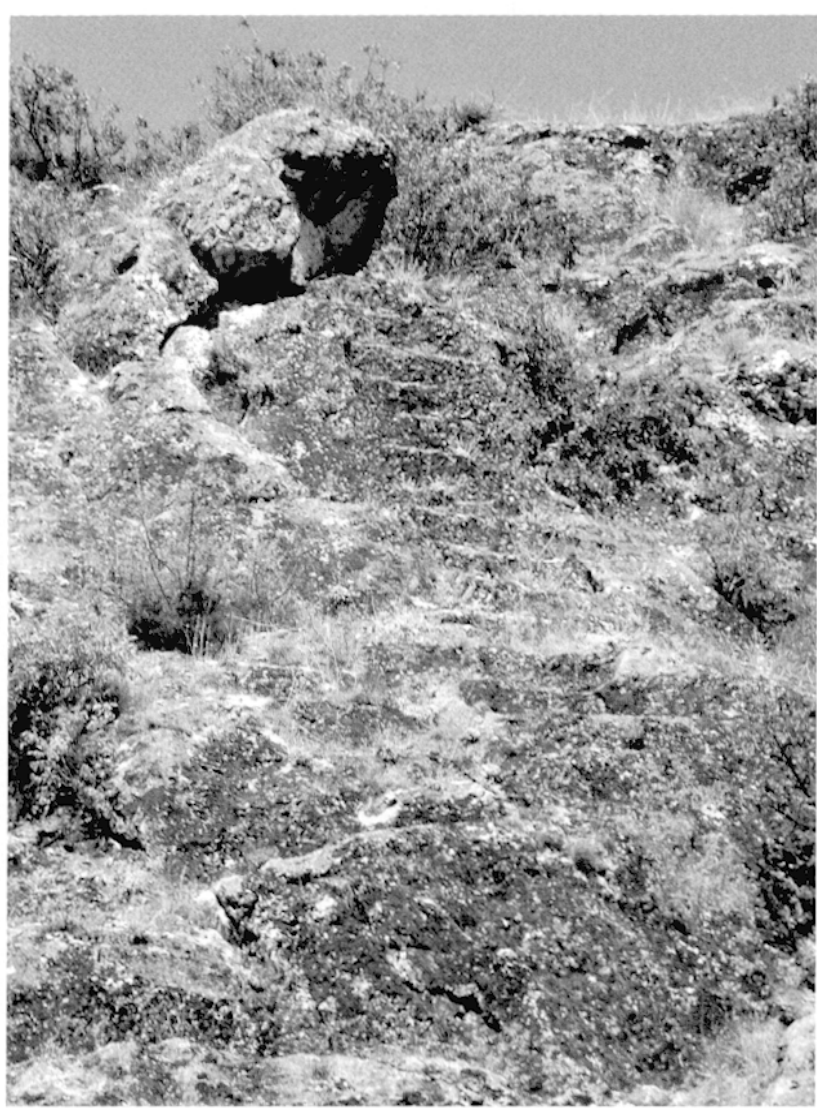

Fig. 16 : Donalar, escaliers.

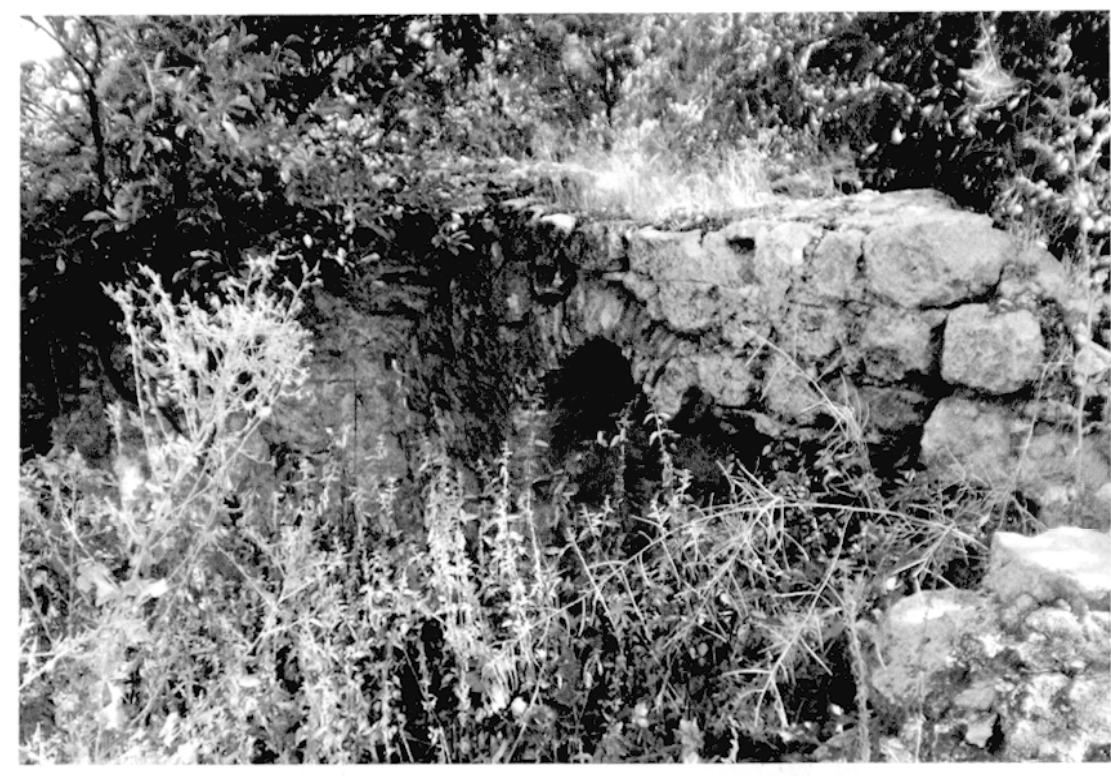

Fig. 17 : Beyköy, thermes d'une uilla ?

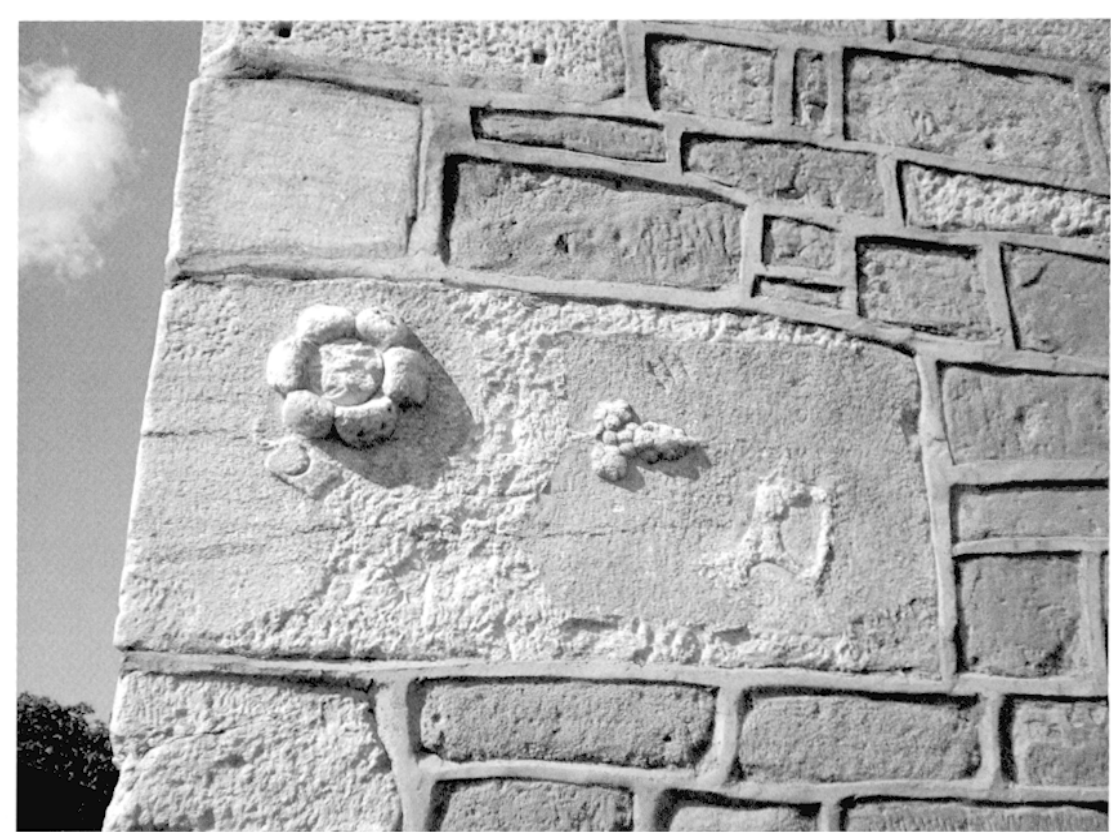

Fig. 18 : Beyköy, bloc monumental orné dans mur de la mosquée la plus ancienne. 
un chapiteau de style ionique, des blocs, dont un mouluré, tous en marbre blanc.

Ces vestiges proviendraient d'un site situé sur un replat naturel de l'autre côté de la route et du lit de l'affluent du Gökırmak, que les villageois appellent Kastellos. Dans les champs, des travaux hydrauliques récents ont mis au jour de gros blocs parallélépipédiques de marbre blanc, un pilastre en marbre blanc (Fig. 19), un autel anépigraphe (Fig. 20) et quelques tegulae. Au vu de la grande taille et de la belle qualité des éléments d'architecture dans le village et dans les champs, des mesures géophysiques effectuées par le Dr. Jörg Faßbinder de l'Université Technique de Munich et de l'étude de l'image satellitaire faisant apparaître un ensemble d'anomalies appartenant à des monuments antiques, il est possible de penser que nous avons ici une agglomération secondaire dépendant de Pompeiopolis ou un sanctuaire extra-urbain.

\subsection{Rive droite du Gökırmak, vallée au Sud de Taşköprü, le long de la route de Çiftlikköy : villages de Kızılkise (zone 20), Kise Köyü, Aptalhasan.}

Dans le village de Kızlkise, peu de fragments sont visibles : une corniche fragmentaire en marbre blanc $(121 \times 37 \times 31 \mathrm{~cm})$ avec perles, pirouettes et cordeau à l'intérieur de l'ancienne mosquée en dehors du village et un pilastre d'angle fragmentaire en marbre blanc $(64 \times 48 \times 14 \mathrm{~cm})$. Le passage dans le village de Kiseköyü a été infructueux car l'ancienne église transformée en mosquée et qui avait donné son nom au village (une contraction de Kilise Köyü) a brûlé en 1979 et la seule colonne subsistant a été transportée au musée de Kastamonu.

Les habitants de Taşköprü nous ont signalé la richesse en vestiges des villages de cette vallée. Une prospection dans le village d'Aptalhasan ou Abdal Hasan Tekkesi Türbesi nous a permis d'inventorier de nombreux blocs, colonnes et chapiteaux en remploi dans la tombe d'un saint musulman (Fig. 22) et dans la cour et les murs de la mosquée (Fig. 21). La présence de vestiges dans ce village laisse supposer une occupation dès la période antique et il apparaît essentiel d'engager une prospection systématique dans les autres villages de la vallée.

\subsection{Zone montagneuse de l'Elek Dağı : village de Meye (zone 22)}

Au Sud du village de Meye, nous avons trouvć deux tombes rupestres. Taillée dans le calcaire, la première est bien conservée (Fig. 23 ; façade : $145 \times 120 \mathrm{~cm}$; porte : $54 \times 42 \mathrm{~cm}$ ). Maçonnée, la seconde est en très mauvais état, car la voûte s'est effondrée à l'occasion de fouilles clandestines. Ce type de sépulture est déjà connu et identifié à Çavuşlar, dans la région de Safranbolu, comme celui adopté par la noblesse sassanide présente en Anatolie centrale dans la seconde moitié du $\mathrm{III}^{\mathrm{e}} \mathrm{s}$. ap. J.-C. ${ }^{24}$.

\subsection{Rive gauche du Gökırmak, au Nord-est de Taşköprü : Kızkalesi et Çarıklı (zone 21)}

De la période byzantine subsistent les vestiges d'une forteresse, qui domine et contrôle la vallée du Gökırmak (Fig. 24 et 25). Les murs du rempart sont constitués de petits moellons, d'assises réglées à joints alternés et d'un mortier de tuileau. A l'intérieur de la forteresse, une imposante citerne a été aménagée. Nous avons remarqué qu'un certain nombre de blocs antiques avaient été remployés dans les fondations d'une des tours (Fig. 26). Ces fragments proviennent vraisemblablement des environs proches ou du village de Çarkklı, où trois fûts de colonnes à astragale sont à l'abandon.

\subsection{Hanönü}

Nous avons terminé cette première prospection du territoire de Pompeiopolis par le site de Hanönü, anciennement Gökçeağaç, car Pascal Fourcade y avait mentionné un caravansérail, peut-être construit sur une église paléochrétienne ${ }^{25}$, ce que $P$. Donceel-Voûte avait infirmé ${ }^{26}$. D'après nos observations, le caravansérail (Fig. 27) a bien été construit à partir d'une église paléochrétienne dont les murs sont relativement bien conservés. Il est possible d'observer à l'intérieur du caravansérail une partie de la nef centrale et des bas-côtés, où des voûtes en briques reposant sur des piliers sont aussi conservées (Fig. 28). A l'extérieur, ont été inventoriés des fragments de fûts de colonnes, de piliers, de consoles qui proviennent vraisemblablement de ce premier édifice paléochrétien. 


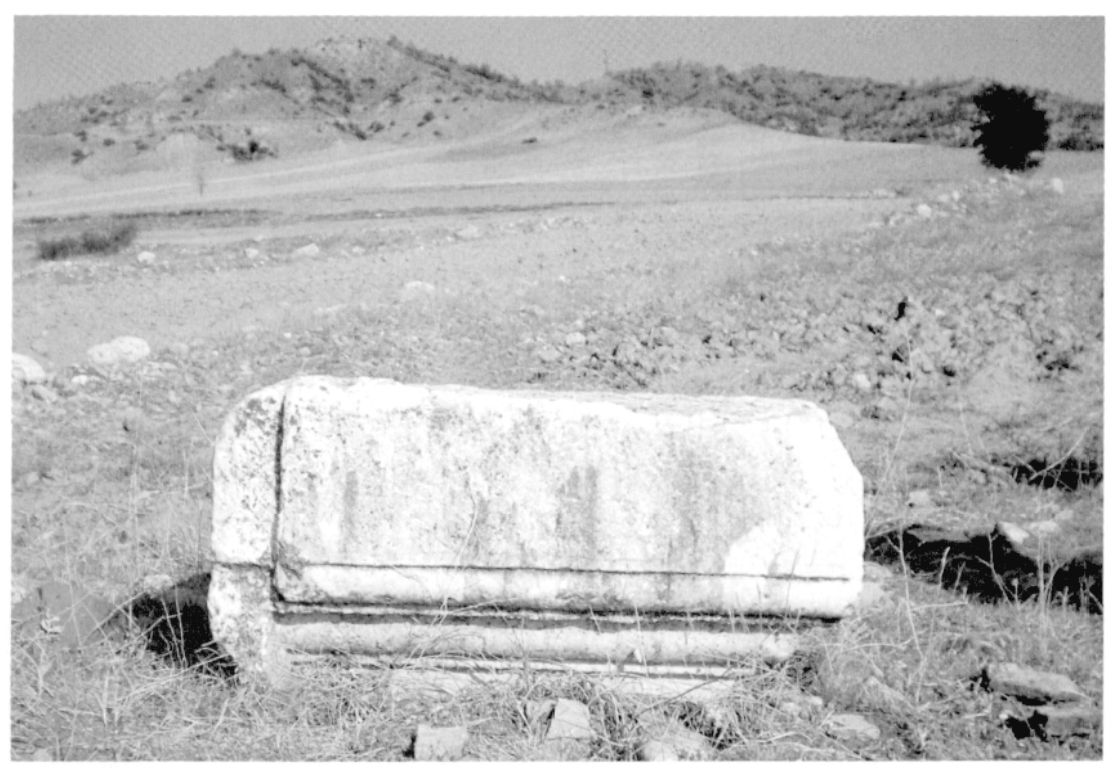

Fig. 19 : Yeniköy, lieu-dit Kastellos, pilastre.

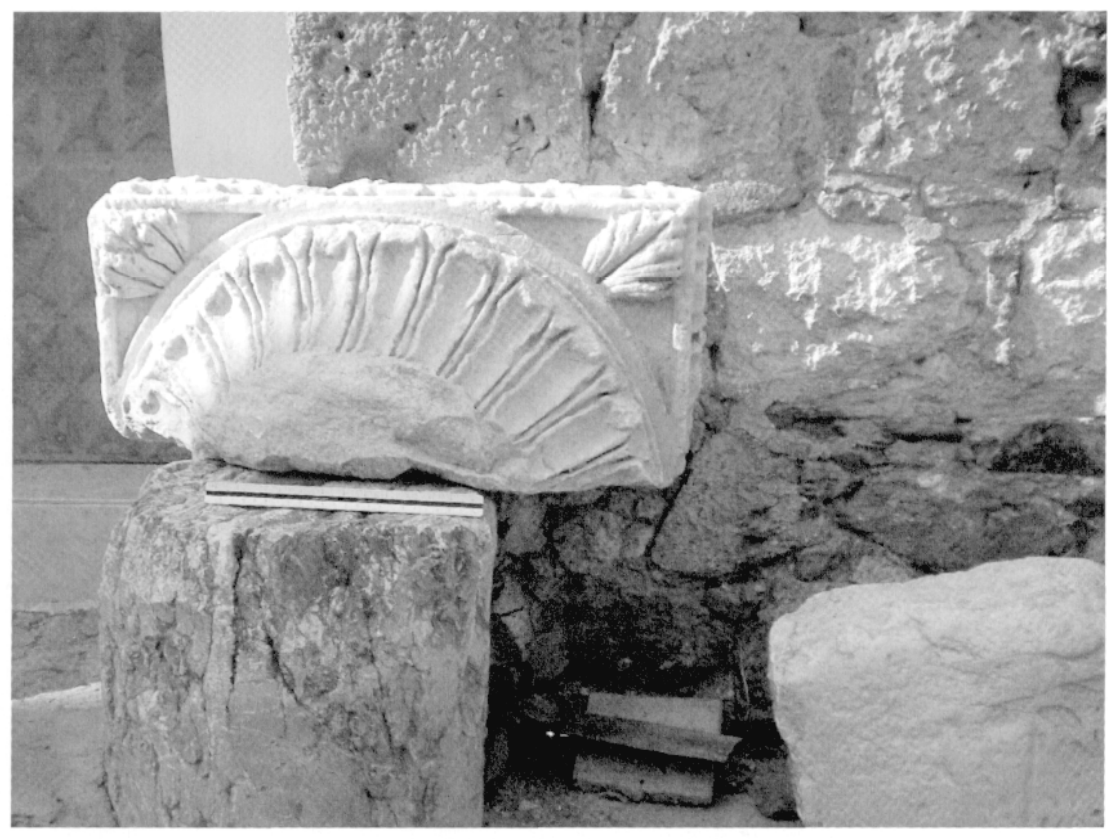

Fig. 21 : Aptalhasan, chapiteau dans la cour de la mosquée.

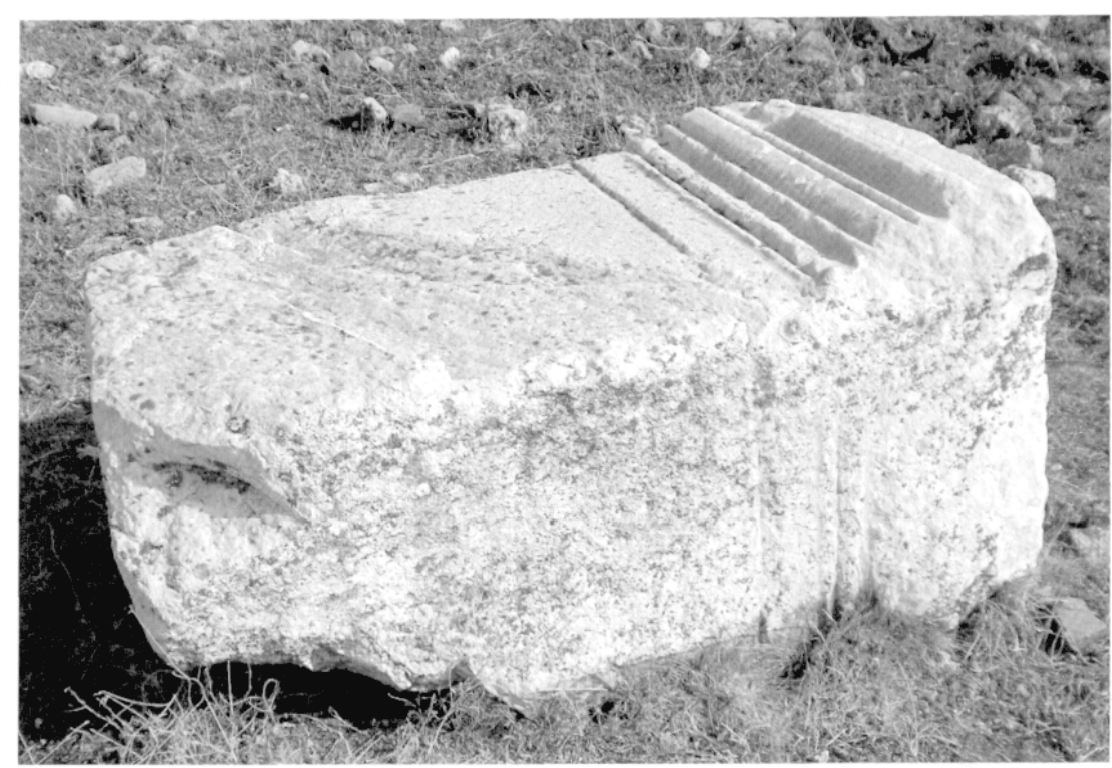

Fig. 20 : Yeniköy, lieu-dit Kastellos, autel anépigraphe.

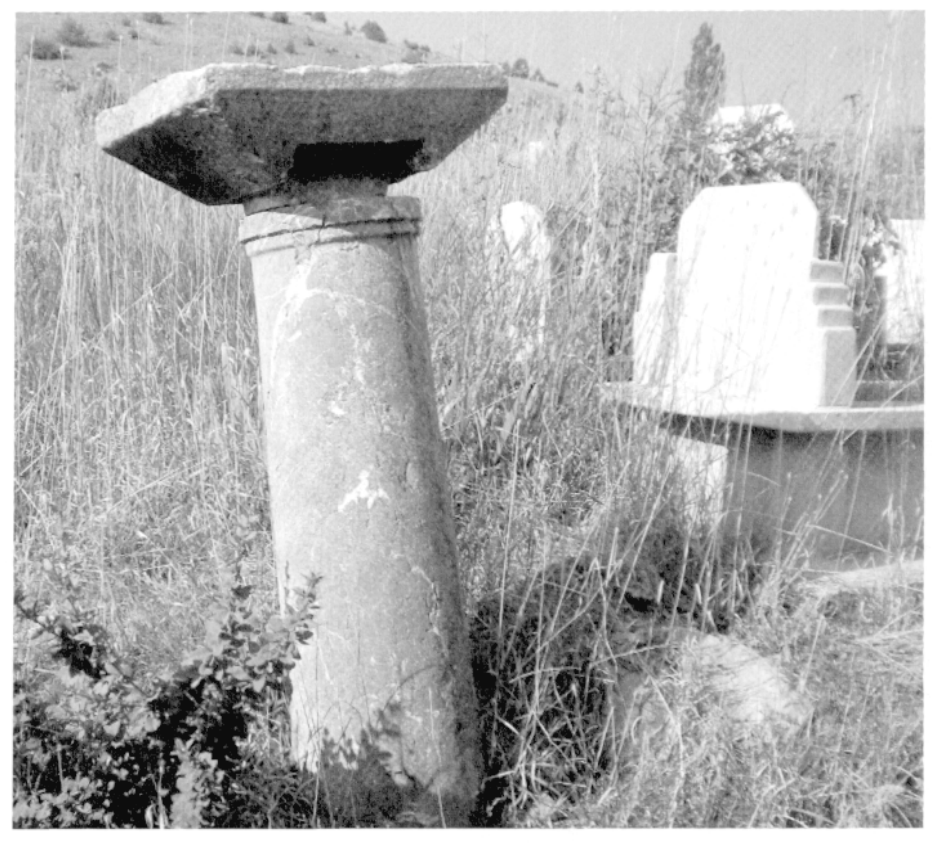

Fig. 22 : Aptalhasan, colonne dans le jardin du türbe. 


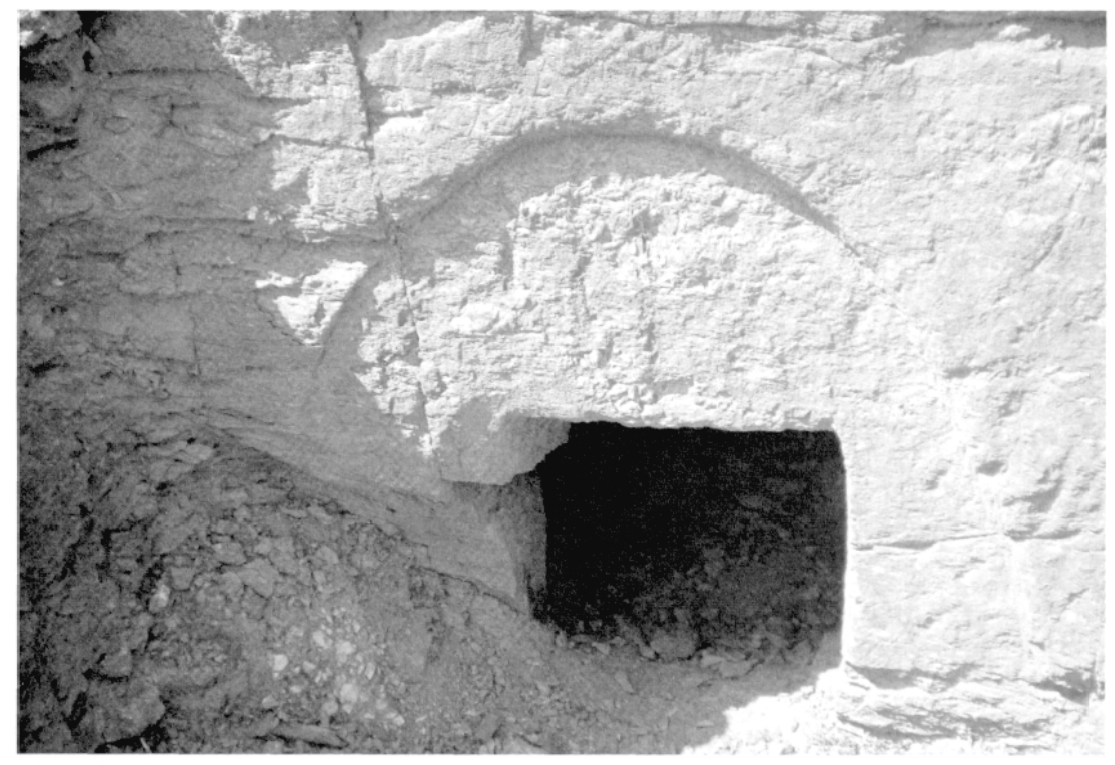

Fig. 23 : Meye, tombe rupestre.

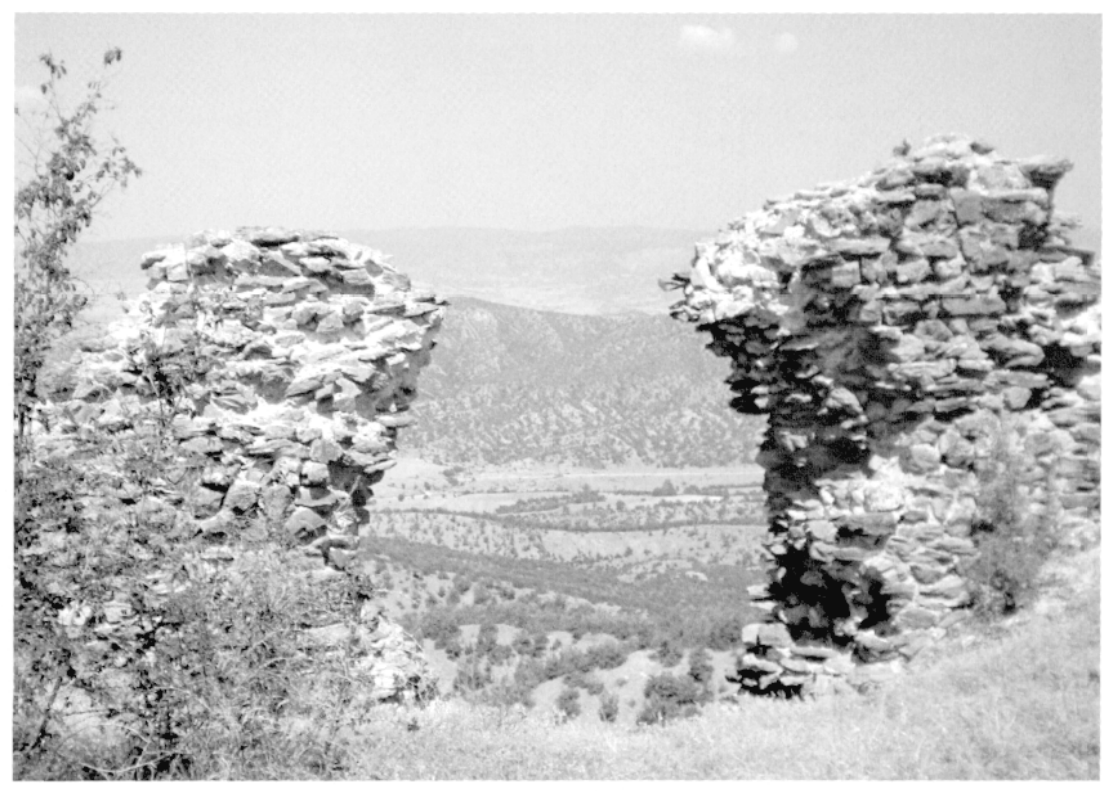

Fig. 24 : Kızkalesi, fortifications.
O⿱丶万⿱⿰㇒一乂

Fig. 25 : Kızkalesi, tour. 


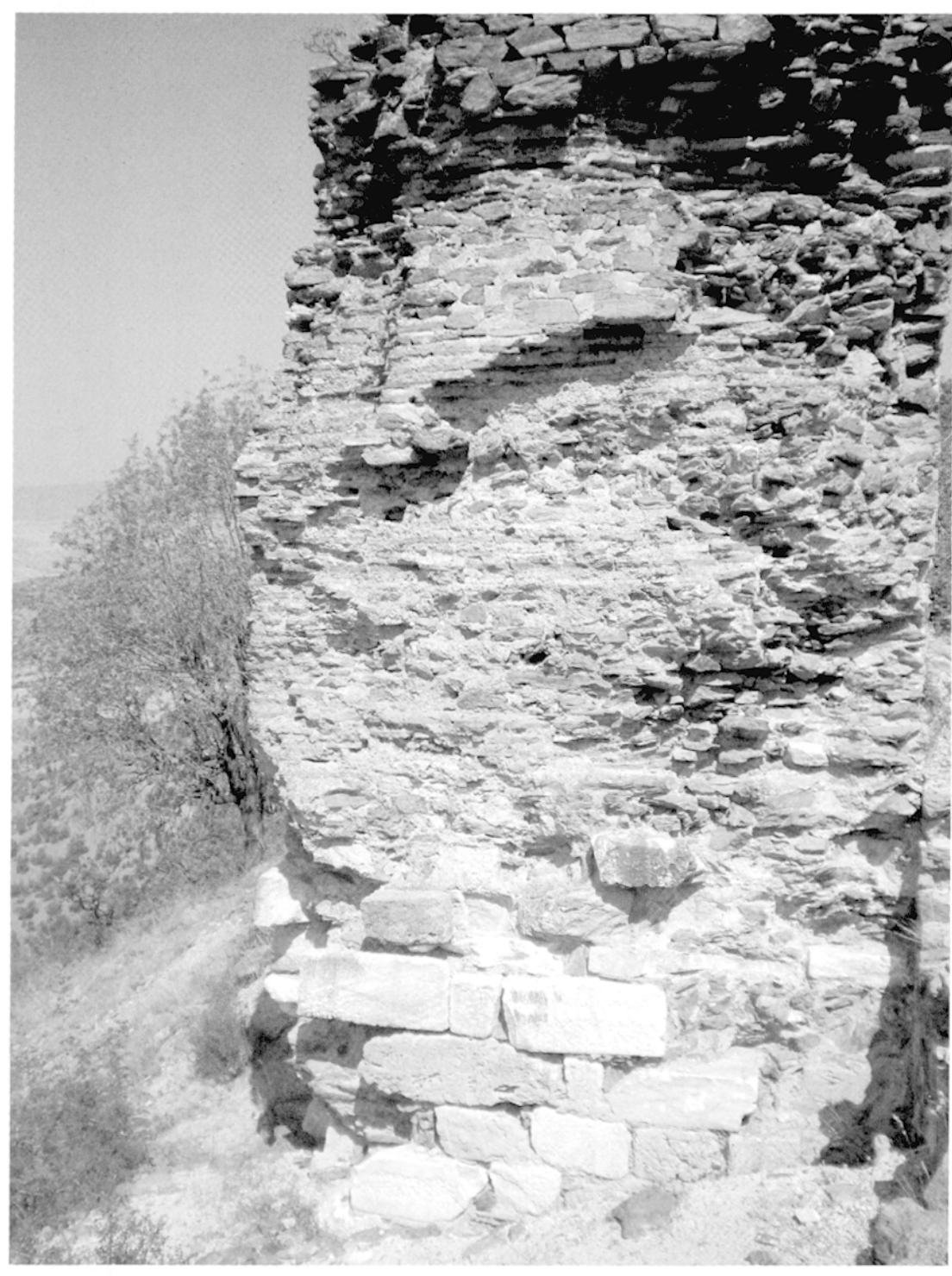

Fig. 26 : Kızkalesi, détail du parement d'une tour.

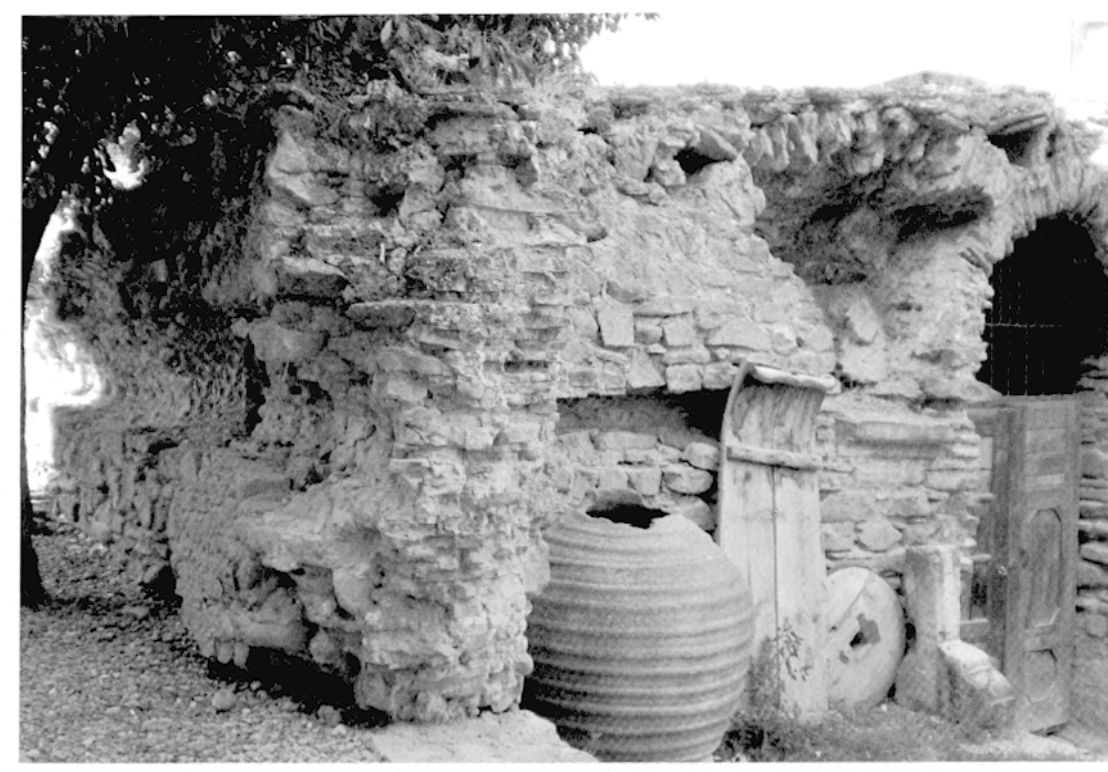

Fig. 27 : Hanönü, extérieur du caravansérail.
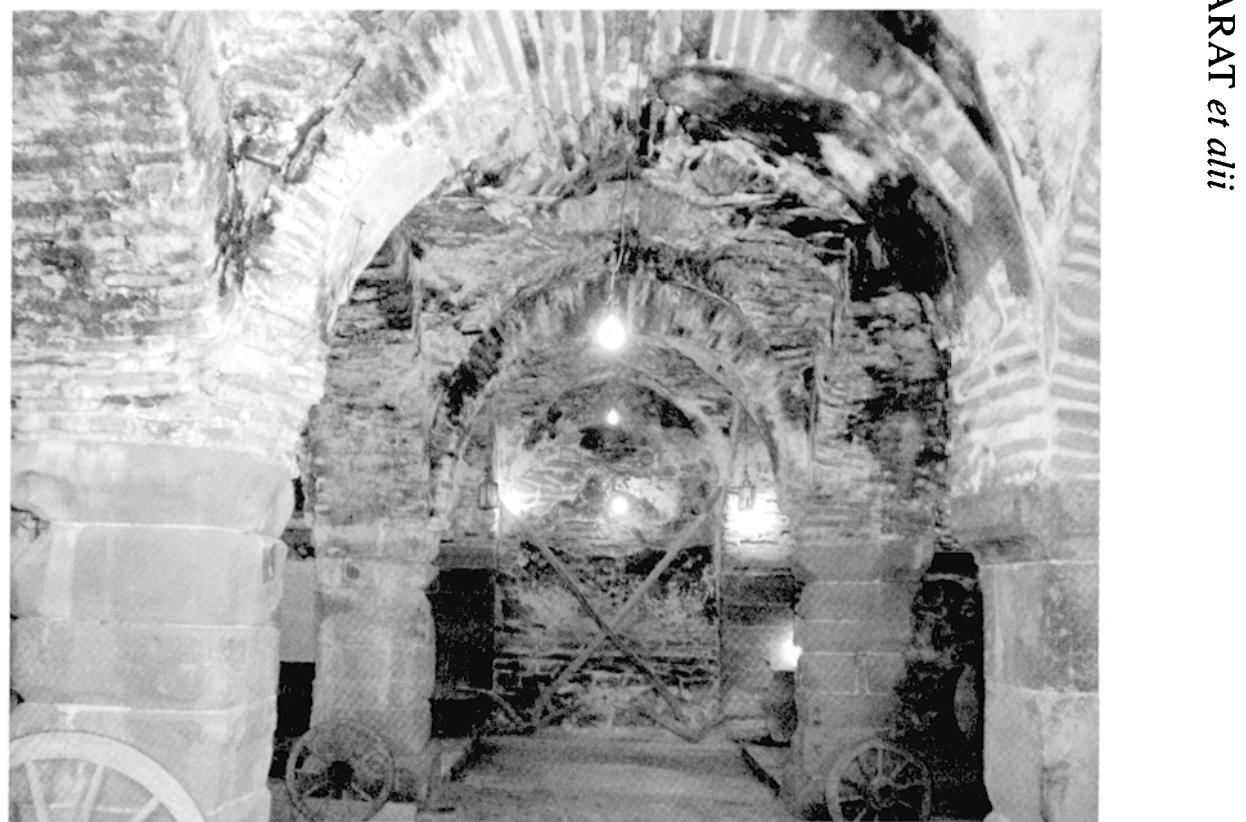

Fig. 28 : Hanönü, intérieur du caravansérail, nef centrale. 


\section{CONCLUSION}

Ce premier travail d'inventaire est un passage obligé pour tous les travaux de recherche à venir sur le territoire de Pompeiopolis et de sa région. Les premiers indices recueillis ne permettent pas actuellement d'avancer des conclusions sur l'occupation du territoire de l'époque archaïque à l'époque romaine. Ce travail doit être poursuivi et étendu par une prospection plus systématique des villages environnants en procédant à des sondages et/ou en recourant à la coopération des physiciens, tenants d'une prospection géophysique.

Les sites et vestiges rencontrés lors de cette première prospection sont d'époque romaine, hormis les tombes rupestres de Bademci et Donalar. La région de Pompeiopolis recèle un fort potentiel archéologique qui ne demande qu'à être pris en compte pour l'étude des occupations paphlagoniennes, achéménides, pontiques et romaines.

C.B. et al.

\section{BIBLIOGRAPHIE}

Barat, C., 2005 : "Voyageurs et perception des vestiges archéologiques à Sinope au temps de la représentation diplomatique française, sous le Consulat et l'Empire", Anabases 2 : 163-175.

Cuinet, V., 1891-1894 [2001] : La Turquie d'Asie. Géographie administrative, statistique, descriptive et raisonnée de chaque province de l'Asie Mineure. VIIL'Anatolie occidentale : Brousse et Castamouni, Paris, réédition Istanbul.

Donceel-Voûte, P., 1979 : "Gökırmak Valley, 1977”, Anatolian Studies 29 : 196-197.

Gall, H. Von, 1966 : Die paphlagonischen Felsgräber, Tübingen.

Fourcade, P., 1811 : "Mémoire sur Pompeiopolis ou Tasch-Koprou, avec quelques remarques sur Tovata ou Voyavat", Annales des voyages, de la géographie et de l'histoire $14: 30-58$.

Leonhard, R., 1915 : Paphlagonia. Reisen und Forschungen im nördlichen Kleinsasien, Berlin.
Marek, Chr., 2008 : "Paphlagonie et Pont. Les tombes rupestres préromaines du nord de l'Anatolie", Les dossiers d'archéologie, 328, juillet/août, 62-71.

Marro, C., Özdoğan A. et Tibet, A., 1996 : "Prospection archéologique franco-turque dans la région de Kastamonu (Mer Noire). Premier rapport préliminaire", Anatolia Antiqua, Eski Anadolu IV : 273-290.

Marro, C., Özdoğan, A., Tibet A., et Kuzucuoğlu, C., 1997 : "Prospection archéologique franco-turque dans la région de Kastamonu (Mer Noire). Deuxième rapport préliminaire", Anatolia Antiqua, Eski Anadolu $\mathrm{V}: 275-306$.

- 1998 : "Prospection archéologique franco-turque dans la région de Kastamonu (Mer Noire). Troisième rapport préliminaire", Anatolia Antiqua, Eski Anadolu VI : 317-335. 